\title{
An Iwasawa-Taniguchi effect for Compton-thick active galactic nuclei
}

\author{
Peter G. Boorman, ${ }^{1 \star}$ Poshak Gandhi, ${ }^{1 \star}$ Mislav Baloković, ${ }^{2,3}$ Murray Brightman, ${ }^{2}$ \\ Fiona Harrison, ${ }^{2}$ Claudio Ricci ${ }^{4,5,6,7}$ and Daniel Stern ${ }^{8}$ \\ ${ }^{1}$ Department of Physics \& Astronomy, Faculty of Physical Sciences and Engineering, University of Southampton, Southampton SO17 1BJ, UK \\ ${ }^{2}$ Cahill Center for Astronomy and Astrophysics, California Institute of Technology, Pasadena, CA 91125, USA \\ ${ }^{3}$ Harvard-Smithsonian Center for Astrophysics, 60 Garden Street, Cambridge, MA 02138, USA \\ ${ }^{4}$ Facultad de Física, Instituto de Astrofísica, Pontificia Universidad Católica de Chile, Casilla 306, Santiago 22, Chile \\ ${ }^{5}$ Núcleo de Astronomía de la Facultad de Ingeniería, Universidad Diego Portales, Av. Ejército Libertador 441, Santiago, Chile \\ ${ }^{6}$ Kavli Institute for Astronomy and Astrophysics, Peking University, Beijing 100871, China \\ ${ }^{7}$ Chinese Academy of Sciences South America Center for Astronomy and China-Chile Joint Center for Astronomy, Camino El Observatorio 1515, Las \\ Condes, Santiago, Chile \\ ${ }^{8}$ Jet Propulsion Laboratory, California Institute of Technology, Pasadena, CA 91109, USA
}

Accepted 2018 March 29. Received 2018 March 29; in original form 2017 September 28

\begin{abstract}
We present the first study of an Iwasawa-Taniguchi/'X-ray Baldwin' effect for Compton-thick active galactic nuclei (AGN). We report a statistically significant anticorrelation between the rest-frame equivalent width (EW) of the narrow core of the neutral $\mathrm{Fe} \mathrm{K} \alpha$ fluorescence emission line, ubiquitously observed in the reflection spectra of obscured AGN, and the mid-infrared $12 \mu \mathrm{m}$ continuum luminosity (taken as a proxy for the bolometric AGN luminosity). Our sample consists of 72 Compton-thick AGN selected from pointed and deep-field observations covering a redshift range of $z \sim 0.0014-3.7$. We employ a Monte Carlo-based fitting method, which returns a Spearman's Rank correlation coefficient of $\rho=-0.28 \pm 0.12$, significant to 98.7 per cent confidence. The best-fitting found is $\log \left(\mathrm{EW}_{\mathrm{Fe} \mathrm{K} \alpha}\right) \propto-0.08 \pm 0.04 \log \left(L_{12 \mu \mathrm{m}}\right)$, which is consistent with multiple studies of the X-ray Baldwin effect for unobscured and mildly obscured AGN. This is an unexpected result, as the $\mathrm{Fe} \mathrm{K} \alpha$ line is conventionally thought to originate from the same region as the underlying reflection continuum, which together constitute the reflection spectrum. We discuss the implications this could have if confirmed on larger samples, including a systematic underestimation of the line-of-sight X-ray obscuring column density and hence the intrinsic luminosities and growth rates for the most luminous AGN.
\end{abstract}

Key words: galaxies: active-infrared: galaxies - X-rays: galaxies.

\section{INTRODUCTION}

X-ray continuum emission from active galactic nuclei (AGN) typically takes the form of a broad-band power law with a highenergy cut-off around $300 \mathrm{keV}$ (Ballantyne 2014; Malizia et al. 2014), and originates from Comptonization of ultraviolet accretion disc photons in a hot X-ray corona (Haardt \& Maraschi 1991, 1993). Line-of-sight opacity alters this emission via photoelectric absorption and Compton scattering. If properly accounted for, this can be used to predict the intrinsic spectral energy distribution of an AGN and thus indirectly study the circumnuclear environment of AGN. Many studies have revealed that the vast majority of AGN are intrinsically obscured with hydrogen column densities $\left(N_{\mathrm{H}}\right)$ greater than the Galactic value (Risaliti, Maiolino \& Salvati

\footnotetext{
^E-mail: p.g.boorman@ soton.ac.uk (PGB); p.gandhi@ soton.ac.uk (PG)
}

1999; Burlon et al. 2011; Ricci et al. 2015, $N_{\mathrm{H}} \gtrsim 10^{22} \mathrm{~cm}^{-2}$ ). For $N_{\mathrm{H}} \lesssim 10^{24} \mathrm{~cm}^{-2}$, the intrinsic power law typically dominates over any other spectral features in the X-ray band. As the column increases to $N_{\mathrm{H}}>1.5 \times 10^{24} \mathrm{~cm}^{-2}$, the obscuring material becomes optically thick in X-rays to Compton scattering, in the Comptonthick regime. Here, the soft X-ray $(E \lesssim 10 \mathrm{keV})$ spectrum is depleted and flattened due to the interplay of photoelectric absorption and Compton downscattering. Depending on the orientation, geometry, and column of the Compton-thick obscurer, the hard Xray spectrum $(E \gtrsim 10 \mathrm{keV})$ can either be dominated by the direct intrinsic power-law component, absorbed along the line of sight (transmission-dominated Compton-thick AGN); or by a Comptonscattered reflection component, from intrinsic flux reprocessed by the obscurer into the line of sight (reflection-dominated Comptonthick AGN).

The geometrical configuration of the X-ray obscuring and reprocessing medium is typically assumed to be roughly axisymmet- 
ric but anisotropic (Ikeda, Awaki \& Terashima 2009; Murphy \& Yaqoob 2009; Brightman \& Nandra 2011; Baloković et al. 2018). This is analogous to the putative torus in the Unified Model of AGN (Antonucci 1993; Urry \& Padovani 1995; Netzer 2015) invoked to explain the infrared and optical emission observed from different classes of AGN as intrinsically a single class observed at different orientation angles. The X-ray obscurer in Compton-thick AGN is what defines the spectral shape of the reprocessed reflection spectrum, which typically features two key components (Lightman \& White 1988; Reynolds 1999):

(i) A narrow $\mathrm{Fe} \mathrm{K} \alpha$ fluorescence emission line arising from neutral (and hence cold) iron, with a characteristic energy of $6.4 \mathrm{keV}$ in the rest frame of the source. This emission line is typically the most prominent in the X-ray spectra of AGN, due to a combination of the fluorescence yield and relative abundances of the gas located within the torus.

(ii) An underlying (flat) Compton scattered continuum with a broad 'Compton hump' peaking at $\sim 30 \mathrm{keV}$ formed from the combination of photoelectric absorption at $E \lesssim 10 \mathrm{keV}$ and Compton downscattering from higher energies.

Modelling the strength and shape of the neutral Fe K $\alpha$ fluorescence line together with the Compton hump can yield the line-ofsight obscuring column to a source. This requires an observed X-ray spectrum spanning the Compton hump at $\sim 30 \mathrm{keV}$ and the soft Xray emission $\lesssim 10 \mathrm{keV}$, to provide constraints on the continuum and reflection components. However, many previous X-ray observations of AGN have typically been restricted to the $E \lesssim 10 \mathrm{keV}$ energy region (Suzaku XIS, Chandra, XMM-Newton), completely missing the Compton hump for local sources. This typically means that any attempt to fit AGN X-ray spectra in this energy region with the objective of constraining the line-of-sight $N_{\mathrm{H}}$ depends heavily on the $\mathrm{Fe} \mathrm{K} \alpha$ fluorescence line alone, and can be uncertain.

Despite being an indicator of high obscuring columns, the equivalent width (EW) of the narrow core of the neutral Fe K $\alpha$ fluorescence line has been observed to anticorrelate with the underlying intrinsic X-ray continuum luminosity in samples of transmissiondominated AGN. This effect was first reported by Iwasawa \& Taniguchi (1993) for a sample of 37 largely unobscured AGN, observed by the Ginga satellite. The best-fitting linear relation derived was of the form $\log \left(\mathrm{EW}_{\mathrm{Fe} \mathrm{K} \alpha}\right) \propto-0.20 \pm 0.03 \log \left(L_{2-10 \mathrm{keV}}\right)$. This is sometimes referred to as the 'X-ray Baldwin' effect due to the similarity with the study by Baldwin (1977) on the anticorrelation between the EW of the $\mathrm{C}$ IV 1549 ultraviolet emission line and AGN continuum. Here, we refer to the X-ray Baldwin effect as the 'Iwasawa-Taniguchi' effect.

The Iwasawa-Taniguchi effect has been explored in further detail for different AGN classes. For example, Page et al. (2004) reported an Iwasawa-Taniguchi effect of $\log \left(\mathrm{EW}_{\mathrm{Fe} \mathrm{K} \alpha}\right) \propto-0.17 \pm$ $0.08 \log \left(L_{2-10 \mathrm{kev}}\right)$ for a sample of 53 type 1 AGN observed by $X M M-N e w t o n$, with the slope being consistent with that of Iwasawa \& Taniguchi. However, Jiang, Wang \& Wang (2006) later reported a much shallower anticorrelation of $\log \left(\mathrm{EW}_{\mathrm{Fe} \mathrm{K} \alpha}\right) \propto$ $-0.10 \pm 0.05 \log \left(L_{2-10 \mathrm{keV}}\right)$ for a sample of 75 radio-quiet AGN observed by XMM-Newton and Chandra. The authors attribute the reduction in slope of the anticorrelation to radio-loud contamination of previous AGN samples, proposing that radio-loud AGN could have an enhanced continuum contribution from a relativistic jet. The authors further postulated that short-term variability of the primary $\mathrm{X}$-ray source could, in part, contribute to the anticorrelation. Despite the shallower gradient found, two measurements of the same gradient would be expected to differ by the separation between Iwasawa
$\&$ Taniguchi and Jiang et al. $\sim 8$ per cent of the time, ${ }^{1}$ and are thus not strongly inconsistent with each other. Bianchi et al. (2007) later studied the Iwasawa-Taniguchi effect for a sample of 157 radio-quiet unobscured type 1 AGN, including narrow line Seyfert 1s (which share some spectral characteristics with obscured AGN). In contrast to Jiang et al. (2006), the authors found a somewhat steeper anticorrelation of $\log \left(\mathrm{EW}_{\mathrm{Fe} \mathrm{K} \alpha}\right) \propto-0.17 \pm 0.03 \log \left(L_{2-10 \mathrm{keV}}\right)$, fully consistent with the original Iwasawa-Taniguchi effect and Page et al. (2004). Bianchi et al. further suggest an additional strong anticorrelation between the $\mathrm{Fe} \mathrm{K} \alpha$ fluorescence line $\mathrm{EW}$ and Eddington ratio. Indeed, Ricci et al. (2013a) tested the positive relation between the photon index and Eddington ratio found for AGN (Lu \& Yu 1999; Shemmer et al. 2006; Risaliti, Young \& Elvis 2009; Brightman et al. 2013; Trakhtenbrot et al. 2017), even into the Compton-thick regime (Brightman et al. 2016), finding that this could contribute to the Iwasawa-Taniguchi effect. This is because a lower Eddington ratio (and thus photon index, resulting in a flatter spectrum) would lead to more photons at the energy required to generate iron $\mathrm{K} \alpha$ fluorescence, giving a larger EW.

Individual source variability has been shown to considerably affect the strength of the anticorrelation, with Shu et al. (2012) finding a reduction in the observed slope from $\log \left(\mathrm{EW}_{\mathrm{Fe} \mathrm{K} \alpha}\right) \propto$ $-0.22 \log \left(L_{2-10 \mathrm{keV}}\right)$ to $\log \left(\mathrm{EW}_{\mathrm{Fe} \mathrm{K} \alpha}\right) \propto-0.13 \log \left(L_{2-10 \mathrm{keV}}\right)$, af ter accounting for the time-averaged $\mathrm{Fe} \mathrm{K} \alpha$ strength in a sample of 32 AGN with $N_{\mathrm{H}} \lesssim 10^{23} \mathrm{~cm}^{-2}$, observed multiple times by the Chandra high-energy grating (HEG).

The conventional Iwasawa-Taniguchi effect describes the strength of the $\mathrm{Fe} \mathrm{K} \alpha$ line relative to the intrinsic continuum (readily available for unobscured AGN), but a difficulty is introduced when trying to study the effect for obscured sources, which by definition start to lack a prominent transmitted intrinsic component in the iron line flux, to measure the EW against. Ricci et al. (2014) report a significant detection of the Iwasawa-Taniguchi effect for two separate samples of Seyfert $1 \mathrm{~s}$ and $2 \mathrm{~s}$, consistently of $\log \left(L_{\mathrm{Fe} \mathrm{K} \alpha} / L_{10-50 \mathrm{keV}}\right) \propto-0.11 \pm 0.01 \log \left(L_{10-50 \mathrm{keV}}\right)$. Type 2 Seyferts are typically observed to be obscured in the optical and often X-rays also (e.g. Koss et al. 2017). Thus, the work of Ricci et al. was the first study into the effect for obscured sources, in which the higher $10-50 \mathrm{keV}$ energy range was used to describe the intrinsic continuum and $\mathrm{Fe} \mathrm{K} \alpha \mathrm{EW}$ since photoelectric absorption is minimized for photons at harder energies. Interestingly, the authors postulate that the consistency of slopes between Seyfert $1 \mathrm{~s}$ and $2 \mathrm{~s}$ could indicate that the physical mechanism responsible for the Iwasawa-Taniguchi effect is unaffected by orientation under Unification schemes. For a breakdown of the results into the Iwasawa-Taniguchi effect from the different works mentioned above, see table 1 of Ricci et al. (2013b).

Numerous physical scenarios have been considered to explain the observed Iwasawa-Taniguchi effect, with one of the most favoured being an intrinsic luminosity-dependent covering factor of neutral obscuring gas surrounding the AGN. This effect was first suggested in Lawrence \& Elvis (1982) \& Lawrence (1991), dubbed the 'receding torus', and has been observed in various large AGN samples. This idea is strengthened by the results from multiple studies reporting an increased number density of obscured AGN at lower X-ray luminosities (Ueda et al. 2011; Lusso et al. 2013; Merloni et al. 2014; Georgakakis et al. 2017). Simulations of torus reprocessing of X-ray emission have also shown that the Fe K $\alpha$ line EW can be dramatically enhanced when the observer is exposed to less intrin-

${ }^{1}$ https://ned.ipac.caltech.edu/level5/Sept01/Orear/frames.html 


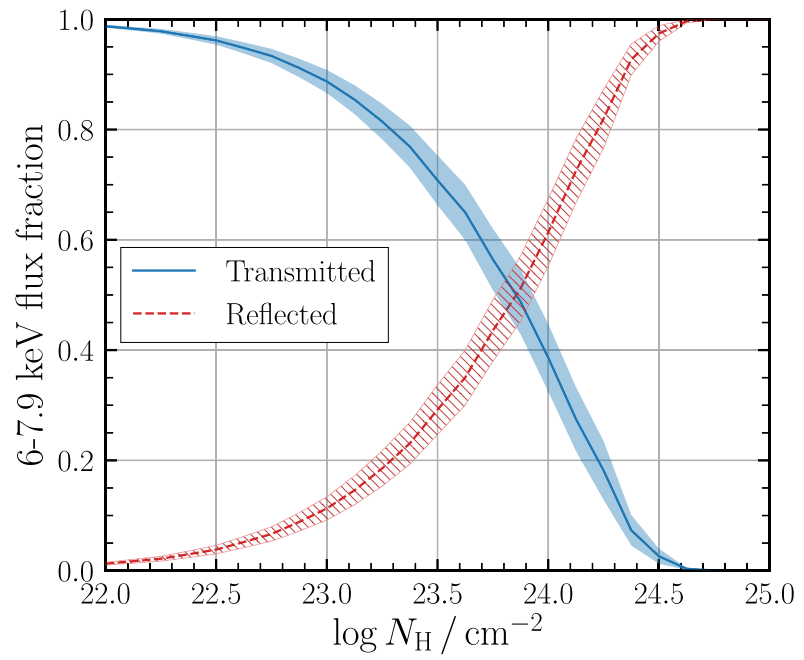

Figure 1. The contribution to the total observed flux in the iron line region (approximated to 6-7.9 $\mathrm{keV}$ ) from the direct transmitted component (blue) and Compton-scattered reflected component (red). This was simulated using the borus 02 X-ray reprocessor model (Baloković et al. 2018), for a spherically distributed obscurer with polar cutouts. For each column density, the average flux ratio for a series of covering factor/inclination angle combinations is plotted with the confidence region showing the range between the minimum and maximum found around the average.

sic flux than the reprocessor (Krolik, Madau \& Zycki 1994), which is physically attained with higher covering factors of the central engine.

A receding torus model provides a possible explanation for the Iwasawa-Taniguchi effect in which the observed spectrum contains a dominant unscattered component, as is the case for transmissiondominated obscured systems. The prominence of the direct transmitted component would scale with intrinsic luminosity, resulting in the narrow $\mathrm{Fe} \mathrm{K} \alpha$ line (arising from the reflection component) being diminished by the brightened intrinsic power law. To illustrate the contribution to the observed flux from the transmitted component versus the reflected component from an anisotropic X-ray reprocessor, Fig. 1 shows the relative contribution to the total line flux (approximated here to $6-7.9 \mathrm{keV}$ ) from the transmitted component (blue) and reflected component (red). This was simulated with the borus02_v170709a (borus02 ${ }^{2}$ ) model (Baloković et al. 2018), in which the obscurer is spherically distributed with polar cutouts. For each column density, we plot the average flux ratio for a series of covering factor/inclination angle combinations, and sources are predicted to become reflection-dominated in the $\mathrm{Fe} \mathrm{K} \alpha$ line for $\log N_{\mathrm{H}} \gtrsim 23.6 \mathrm{~cm}^{-2}$.

Recent dedicated studies into specific X-ray-obscured AGN appear to show a trend of decreased neutral $\mathrm{Fe} \mathrm{K} \alpha$ line EW, with increasing luminosity. Here, we highlight three Compton-thick case studies for comparison; also illustrated in Fig. 2:

(1) Local low-luminosity Compton-thick Seyferts typically show prominent lines. One of the strongest observed $\mathrm{Fe} \mathrm{K} \alpha$ line EWs found to date was for IC 3639 (Boorman et al. 2016); a reflectiondominated Compton-thick AGN with infrared bolometric luminosity (in the 8-1000 $\mu \mathrm{m}$ wavelength range) of $\log \left(L_{8-1000 \mu \mathrm{m}}\left[L_{\odot}\right]\right) \sim$ 10.9 and $\mathrm{EW}_{\mathrm{Fe} \mathrm{K} \alpha} \sim 3 \mathrm{keV}$, relative to the observed underlying reflection continuum.

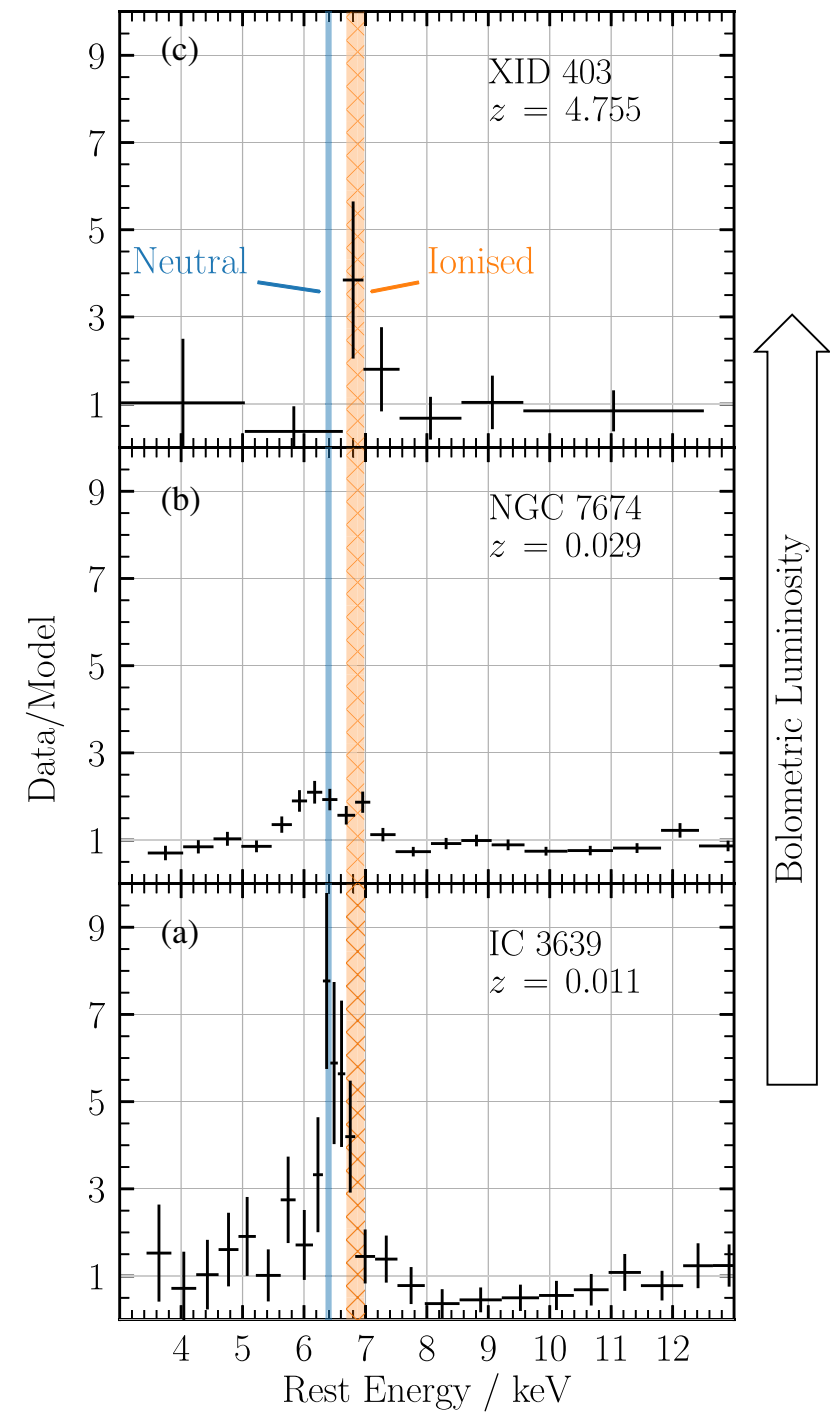

Figure 2. Three Compton-thick case studies illustrating the motivation for the studying a Compton-thick Iwasawa-Taniguchi effect. Panel (a): IC 3639 (Boorman et al. 2016): a local Compton-thick AGN at $z=0.011$ with bolometric infrared luminosity, $\log \left(L_{8-1000 \mu \mathrm{m}}\left[L_{\odot}\right]\right) \sim 10.9$, and one of the strongest neutral Fe K $\alpha$ lines reported in the literature. Panel (b): NGC 7674 (Gandhi et al. 2017): a local Compton-thick AGN, and luminous infrared galaxy with bolometric infrared luminosity, $\log \left(L_{8-1000 \mu \mathrm{m}}\left[L_{\odot}\right]\right) \sim 11.6$. Contrastingly, this source has the lowest neutral Fe $\mathrm{K} \alpha \mathrm{EW}$ reported for local Compton-thick AGN. Furthermore, the spectrum clearly shows a contribution to the residuals in the ionized energy region. Panel (c): LESS J033229.4-275619 (Gilli et al. 2014): the highest redshift Compton-thick AGN currently known, with $\log \left(L_{8-1000 \mu \mathrm{m}}\left[L_{\odot}\right]\right) \sim 12.8$. The spectrum plotted is from a $7 \mathrm{Ms}$ observation of CDFS. The neutral emission is not detected, yet a considerable flux contribution can be seen in the ionized emission line energy region. Each source was fitted with a redshifted power law in xspec, and the resulting data/model ratio is shown. For all panels, the narrow core of the neutral line is shown with the orange region for $E=6.35-6.45 \mathrm{keV}$, and the ionized line region is shown in blue hatch for the $E=6.69-6.98 \mathrm{keV}$ region (to encompass the 6.7 and $6.97 \mathrm{keV}$ ionized lines).

\footnotetext{
${ }^{2}$ Available at http://www.astro.caltech.edu/ mislavb/download/index.html
} 
(2) On the other hand, NGC 7674 (Gandhi et al. 2017) is a heavily Compton-thick Seyfert 2, with a higher infrared bolometric luminosity of $\log \left(L_{8-1000 \mu \mathrm{m}}\left[L_{\odot}\right]\right) \sim 11.6$. Yet the source has an observed EW of the neutral line of $\mathrm{EW}_{\mathrm{Fe} \alpha} \sim 0.4 \mathrm{keV}$ : the lowest constrained $\mathrm{EW}$ of the $\mathrm{Fe} \mathrm{K} \alpha$ line detected for any bona-fide Compton-thick AGN to date.

(3) At the highest luminosities, Gilli et al. (2011, 2014, LESS J033229.4-275619) is the most distant $(z \sim 4.75)$ Comptonthick AGN classified to date, with infrared bolometric luminosity $\log \left(L_{8-1000 \mu \mathrm{m}}\left[L_{\odot}\right]\right) \sim 12.8$. Interestingly, the neutral Fe K $\alpha$ fluorescence line is not detected in the observed X-ray spectrum obtained from the $4 \mathrm{Ms}$ Chandra Deep Field South (CDFS) observation, yet with a prominent ionized Hydrogen-like iron line at $\sim 6.9 \mathrm{keV}$ to $\sim 2 \sigma$ confidence, with rest-frame $\mathrm{EW}=2.8_{-1.4}^{+1.7} \mathrm{keV}$. In fact, there is increasing observational evidence for prominent ionized iron lines in luminous infrared galaxies (LIRGs: $\log \left(L_{8-1000 \mu \mathrm{m}}\left[L_{\odot}\right]\right)>11$; Iwasawa et al. 2009).

We note that although the contribution to the infrared flux from star formation will increase with bolometric flux, the AGN contribution also increases. This means a higher infrared flux should indicate a more intrinsically luminous AGN. These three case study sources are illustrated in Fig. 2, in which we plot the data/model ratio for each source after fitting a power law to the observed spectrum. Although NGC 7674 appears to show a large component to the observed flux around 6-6.5 keV, the narrow core of the neutral $\mathrm{Fe} \mathrm{K} \alpha$ line is considerably weaker. The panels have been binned for clarity.

This paper presents the first study into an Iwasawa-Taniguchi effect for Compton-thick AGN, with $\mathrm{Fe} \mathrm{K} \alpha \mathrm{EW}$ s measured relative to the observed continuum versus rest-frame mid-infrared $12 \mu \mathrm{m}$ luminosity $\left(L_{12 \mu \mathrm{m}}\right.$; taken as a proxy for the intrinsic AGN bolometric luminosity). The cosmology adopted for computing luminosity distances is $H_{0}=67.3 \mathrm{~km} \mathrm{~s}^{-1} \mathrm{Mpc}^{-1}, \Omega_{\Lambda}=0.685$, and $\Omega_{\mathrm{M}}=0.315$ (Planck Collaboration XVI 2014). ${ }^{3}$ The paper is organized as follows: Section 2 describes our source selection and the sample used in our statistical analysis. Section 3 then describes our method for clarifying candidate Compton-thick AGN, as well as for determining the $L_{12 \mu \mathrm{m}}$ and $\mathrm{Fe} \mathrm{K} \alpha \mathrm{EW}$ values. We then discuss our fitting procedure. Section 4 comprises our main results, followed by the discussion and implications of the effect if confirmed on future larger Compton-thick AGN samples, in Section 5. We summarize our findings in Section 6.

\section{THE SAMPLE}

Our primary goal whilst collating Compton-thick candidates from the literature was to cover a broad redshift (and hence luminosity) range. Furthermore, X-ray spectra encompassing the observed frame neutral $\mathrm{Fe} \mathrm{K} \alpha$ fluorescence line, seen at $6.4 /(1+z) \mathrm{keV}$ in the rest frame, were required. In order to robustly quantify, the EW required a detection of the underlying observed continuum, neighbouring the line centroid. Below we give details of the high- and low-redshift subsamples we have included in our work.

\footnotetext{
${ }^{3}$ Redshift-dependent distances are used for consistency across the full sample. Only a handful of the closest AGN have redshift-independent distances which scatter around our adopted luminosity distances.
}

\subsection{High redshift}

For higher redshift (or fainter) sources, Chandra observations were ideal due to low background and optimal sensitivities in the $0.5-8.0 \mathrm{keV}$ energy range. At high redshift, the $k$-corrected Compton hump also shifts to the observed Chandra energy range. A considerable contribution to our sample thus includes the Brightman et al. (2014) compilation of Compton-thick AGN candidates collated from archival deep Chandra surveys. The original sample includes $\sim 100$ Compton-thick candidates. A source was only retained for our study if it met the following criteria:

(i) $>50$ total X-ray counts detected in the Chandra energy band.

(ii) A spectroscopic redshift.

(iii) A line-of-sight column density of $N_{\mathrm{H}} \geq 1.5 \times 10^{24} \mathrm{~cm}^{-2}$ at 90 per cent confidence, determined by Brightman et al. (2014).

(iv) Infrared detection by the Wide-field Infrared Survey Explorer (WISE) ${ }^{4}$ or Spitzer Space Telescope to enable a reliable $L_{12 \mu \mathrm{m}}$ estimate.

Of the resulting candidates, a further two were excluded due to a disagreement with our Compton-thick classification (COSMOS 0661 and COSMOS 1517: Section 3), leaving a total of 27 sources from Brightman et al. (2014). An additional five highredshift sources come from further Compton-thick studies by Feruglio et al. (2011), Corral et al. (2016, BzK 4892), Georgantopoulos et al. (2013, XMMID 324), Lanzuisi et al. (2015, XMMID 2608, XMMID 60152), and Hlavacek-Larrondo et al. (2017, IRAS F15307+3252). In total, 32 sources make up our high-redshift subsample of Compton-thick candidates.

\subsection{Low redshift}

A major contribution to our low-redshift subsample comes from Ricci et al. (2015). The sample consists of 55 Compton-thick AGN candidates selected from the Neil Gehrels Swift/Burst Alert Telescope (BAT) 70-month catalogue, all within the local Universe (average $z=0.055)$. Of these 55 , we rejected 19 sources without publicly available NuSTAR observations. NuSTAR (Harrison et al. 2013) is the first true hard X-ray imaging instrument in the $3-79 \mathrm{keV}$ energy range, encompassing the full underlying reflection continuum for low-redshift AGN, and thus ideal for studying Compton-thick candidates. By combining with soft X-ray observations, many works have constrained the $N_{\mathrm{H}}$ values for numerous obscured, Comptonthick and changing-look AGN to date (e.g. Arévalo et al. 2014, Circinus Galaxy; Baloković et al. 2014, NGC 424, NGC 1320, IC 2560; Gandhi et al. 2014, Mrk 34; Teng et al. 2014, Mrk 231; Annuar et al. 2015, NGC 5643; Bauer et al. 2015, NGC 1068; Ptak et al. 2015, Arp 299; Boorman et al. 2016, IC 3639; Megamaser sample; Masini et al. 2016, Mrk 1210; Ricci et al. 2016, IC 751; Ricci et al. 2017, WISE J1036+0449; Annuar et al. 2017, NGC 1448; Gandhi et al. 2017, NGC 7674), hence our preference for NUSTAR availability.

An additional three sources from the Ricci et al. (2015) sample were excluded due to a disagreement with our mid-infrared diagnostic Compton-thick classification (2MASX J09235371-3141305; MCG -02-12-017; NGC 6232, Section 3).

The last contribution to our low-redshift subsample comes from the Gandhi et al. (2014) compilation of bona-fide Compton-thick

${ }^{4}$ A 'reliable' WISE detection corresponds to a detection with $\mathrm{S} / \mathrm{N}>5$. See ht tp://wise2.ipac.caltech.edu/docs/release/allsky/expsup/sec5_3.html for further details. 
AGN, updated to include IC 3639 (Boorman et al. 2016), NGC 1448 (Annuar et al. 2017), and NGC 7674 (Gandhi et al. 2017), whilst excluding changing-look candidates: Mrk 3 (Ricci et al. 2015, find a Compton-thin column density to 90 per cent confidence), NGC 4102, NGC 4939, ${ }^{5}$ NGC 4785 (Gandhi et al. 2015a; Marchesi et al. 2017), and NGC 7582 (Rivers et al. 2015). In total, 40 sources make up our low-redshift subsample of Compton-thick candidates. Full details of the 72 (low + high redshift) Compton-thick candidates in our sample are included in Table 1.

\section{METHOD}

\subsection{Infrared luminosities}

In selecting a suitable proxy for the bolometric luminosity of each source, we adhered to the following criteria: (1) the bolometric luminosity could neither be derived from the spectral energy region responsible for the neutral $\mathrm{Fe} \mathrm{K} \alpha$ line nor from the continuum surrounding the line that would be used to derive an EW and (2) the proxy should be prominent and well detected for Compton-thick AGN.

We used the infrared contribution to the broad-band spectra of our AGN sample, which is considered to have sizeable contributions in this wavelength range due to reprocessing of the primary intrinsic AGN emission. Since typical AGN contributions to composite galaxy spectra dominate at $\sim 6-20 \mu \mathrm{m}$ (Mullaney et al. 2011), we used the rest-frame $12 \mu \mathrm{m}$ luminosity of each source.

To determine the rest-frame $12 \mu \mathrm{m}$ luminosity, we utilized the infrared spectral template of Mullaney et al. (2011) to interpolate the rest-frame $12 \mu \mathrm{m}$ flux from observed-frame flux measurements as close to $12 \mu \mathrm{m}$ as possible. For high-quality infrared observations, we use the WISE and Spitzer Multiband Imaging Photometer (MIPS). WISE had four imaging channels onboard (W1, W2, $W 3$, and $W 4$ ) corresponding to $\lambda=3.35,4.60,11.56,22.09 \mu \mathrm{m}$, respectively (Wright et al. 2010), whereas Spitzer/MIPS was capable of imaging in spectral bands centred on $\lambda=24,70,160 \mu \mathrm{m}$. For $z \lesssim 0.84$ a robust interpolation could be made from $W 3$ and W4 observations. However, for higher redshift sources in which the $k$-correction shifts the rest-frame $12 \mu \mathrm{m}$ luminosity to wavelengths beyond W4 (or for poorly constrained/faint observations from WISE), we use Spitzer/MIPS.

For archival WISE observations, we use the AllWISE Source Catalog ${ }^{6}$ to get profile-fitted magnitudes and the NASA Extragalactic Database (NED) ${ }^{7}$ to search for archival Spitzer/MIPS observations.

To test how representative the Mullaney et al. (2011) template was for predicting the $12 \mu \mathrm{m}$ luminosity for the AGN in our sample with $L_{2-10 \mathrm{keV}}<10^{42} \mathrm{erg} \mathrm{s}^{-1}$ or $L_{2-10 \mathrm{keV}}>10^{44} \mathrm{erg} \mathrm{s}^{-1}$, we compared the interpolated luminosities with those predicted from the type 2 AGN template from Polletta et al. (2007), which were derived over a wider range of luminosities. On average, the offset between the interpolated luminosities from the two templates was only $\sim 0.06$ dex.

\footnotetext{
${ }^{5}$ Our own analysis of the archival XMM-Newton EPIC/PN spectrum as compared to the more recent NUSTAR FPMA \& FPMB spectra strongly indicate a changing-look AGN for these sources.

${ }^{6} \mathrm{http} / / /$ irsa.ipac.caltech.edu/cgi-bin/Gator/nph-dd

${ }^{7}$ http://ned.ipac.caltech.edu
}

\subsection{Compton-thick confirmation of sample}

Strong correlations between mid-infrared and intrinsic X-ray emission have been found with ground-based high-angular-resolution observations of AGN, around $12 \mu \mathrm{m}$ (Horst et al. 2008; Gandhi et al. 2009; Levenson et al. 2009; Asmus et al. 2015). A similar correlation has been found as a function of large aperture $6 \mu \mathrm{m}$ luminosity, with akin results (Lutz et al. 2004; Mateos et al. 2015; Stern 2015; Chen et al. 2017), and at $5.8 \mu \mathrm{m}$ (Lanzuisi et al. 2009). The $12 \mu \mathrm{m}$ luminosity correlation has been used with considerable success for identifying candidate Compton-thick AGN. Correcting $\mathrm{X}$-ray absorption in Compton-thick sources acts to increase the observed X-ray luminosity to values consistent with the relation. We refer the reader to Boorman et al. (2016) for the effects of absorption correction on X-ray luminosities relative to their observed mid-infrared luminosities for the Gandhi et al. (2014) compilation of bona fide Compton-thick AGN. Here, we use the study of the $\mathrm{X}$-ray versus $12 \mu \mathrm{m}$ correlation reported in Asmus et al. (2015) to classify our sample as candidate Compton-thick.

The rest-frame observed (i.e. absorbed) $2-10 \mathrm{keV}$ luminosity was computed from a fit to the available X-ray spectra within XSPEC (for objects without a reported observed X-ray flux), and plotted against the rest-frame $12 \mu \mathrm{m}$ luminosity, interpolated from the Mullaney et al. (2011) AGN spectral template. These observed fluxes are plotted in Fig. 3 (grey points), with a 30 per cent and 15 percent uncertainty on the X-ray and $12 \mu \mathrm{m}$ luminosities, respectively. The original correlation found by Asmus et al. (2015) is shown with a solid (green) line for clarity, together with the $1 \sigma$ scatter. On average, the sample displays a mean ratio of observed X-ray to midinfrared flux of $-2.0 \pm 0.7 \mathrm{dex}$, and this is shown over plotted with a dashed (grey) line and shading. An average deviation of greater than two orders of magnitude from the relation is indicative of Comptonthick levels of obscuration found in previous works. However, from this relation, 2MASX J09235371-3141305, MCG -02-12-017, NGC 6232, COSMOS 0661, and COSMOS 1517 displayed midinfrared fluxes that agreed with the observed X-ray flux within the uncertainties found by Asmus et al. (2015). This could suggest that the observed X-ray flux has a major contribution from the transmitted component, i.e. is only partially obscured and thus were excluded from our Compton-thick sample.

\subsubsection{Star formation contamination of $L_{12 \mu \mathrm{m}}$}

To test for infrared star formation contamination, we first used the $12 \mu \mathrm{m}$ observations from Asmus et al. (2014). This work minimized star formation contamination in measuring mid-infrared fluxes of local sources by using high-angular-resolution $(\lesssim 0.4$ arcsec) imaging with ground-based $8 \mathrm{~m}$ class telescopes. Such contamination would not be excluded from WISE-based measurements, that were used in our sample for these sources, due to the larger angular resolution (FWHM) of 6.1, 6.4, 6.5, and 12.0 arcsec for $W 1, W 2$, $W 3$, and $W 4$, respectively. Sixteen of our sample of 72 sources have measured fluxes in Asmus et al. (2014). The average X-ray to mid-infrared flux ratio for these 16 sources was consistent with the equivalent ratio for the full sample. To fully account for this in the remainder of our sample without high-angular-resolution measurements, we conservatively use the average change in flux between WISE and Asmus et al. (0.29 dex) added in quadrature to the original 15 percent uncertainty assigned to the template interpolated flux as the lower error bar for all sources lacking a mid-infrared observation from Asmus et al. (2014), giving 0.30 dex. For the 16 
Table 1. Complete list of all sources used in determining the anticorrelation plotted in Fig. 4, ordered by rest-frame $12 \mu \mathrm{m}$ luminosity. Information on specific columns is as follows: (1) ID specific to each source, corresponding to the index featured on plots throughout the paper. (2) Source name. (3) Spectroscopic redshift. (4) Right ascension in degrees. (5) Declination in degrees. (6) Source reference. (7) Logarithm of the observed rest-frame 2-10 keV luminosity, measured in $\mathrm{erg} \mathrm{s}^{-1}$. (8) Logarithm of the rest-frame $12 \mu \mathrm{m}$ luminosity predicted from the Mullaney et al. (2011) infrared spectral template. (9) Rest-frame neutral $\mathrm{Fe} \mathrm{K} \alpha$ fluorescence line EW measured in $\mathrm{keV}$. (10) and (11) Upper and lower limits on the rest-frame neutral Fe Ka fluorescence line EW measured in $\mathrm{keV}$ (values of 0 and -1 , respectively, denote an upper limit to EW). (12) Reference used for EW value. References: Feruglio et al. (2011, Fer11), Goulding et al. (2012, Gou12), Georgantopoulos et al. (2013, Geo13), Brightman et al. (2014, Br14), Gandhi et al. (2014, Gan14), Baloković et al. (2014, Bal14), Lanzuisi et al. (2015, Lan15), Ricci et al. (2015, Ric15), Ptak et al. (2015, Pta15), Masini et al. (2017, Mas16), Corral et al. (2016, Cor16), Gandhi et al. (2017, Gan17), Hlavacek-Larrondo et al. (2017, H-L17), Annuar et al. (2017, Ann17), Boo18: this work.

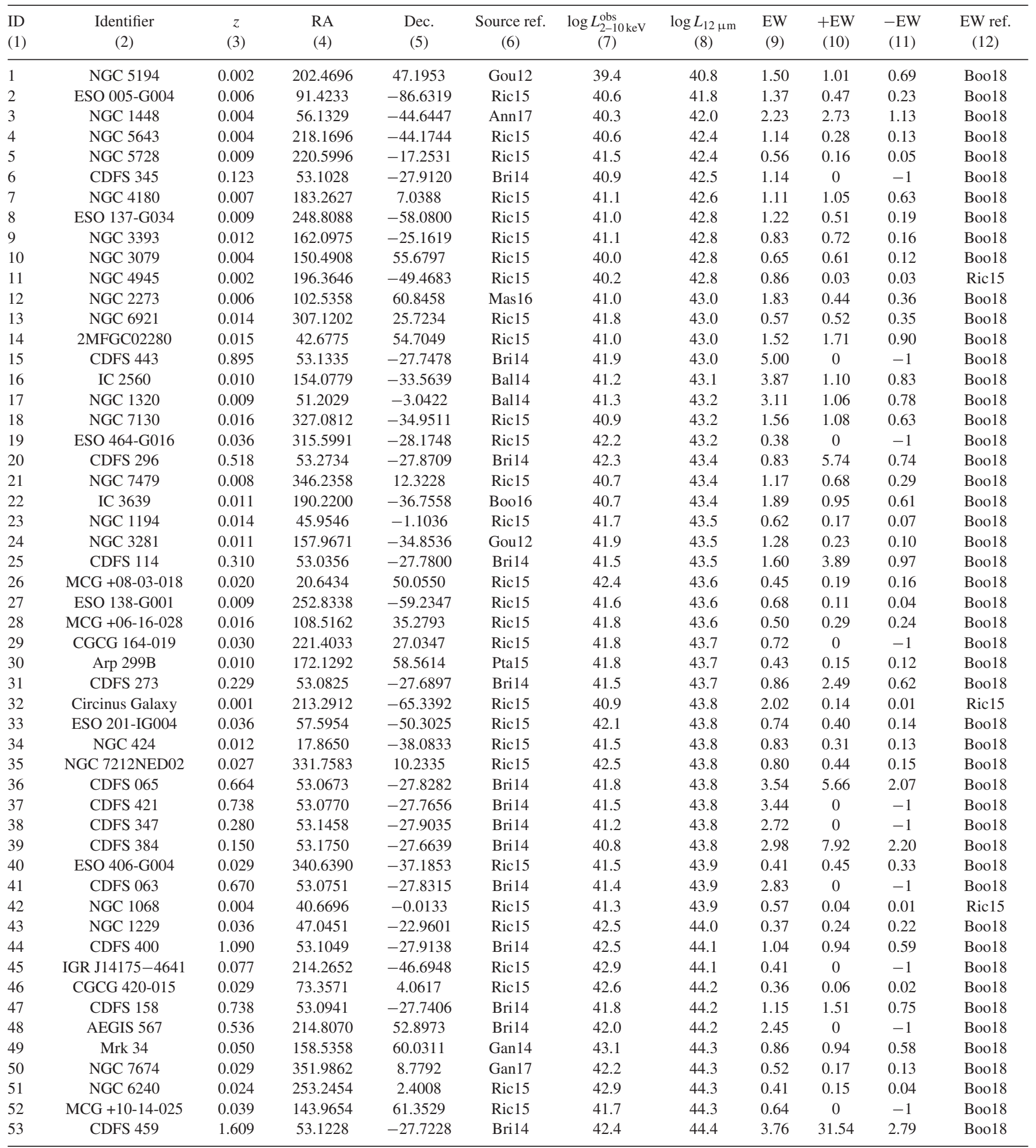


Table 1 - continued

\begin{tabular}{|c|c|c|c|c|c|c|c|c|c|c|c|}
\hline $\begin{array}{l}\text { ID } \\
(1)\end{array}$ & $\begin{array}{l}\text { Identifier } \\
\text { (2) }\end{array}$ & $\begin{array}{c}z \\
(3)\end{array}$ & $\begin{array}{l}\text { RA } \\
(4)\end{array}$ & $\begin{array}{l}\text { Dec. } \\
(5)\end{array}$ & $\begin{array}{l}\text { Source ref. } \\
\text { (6) }\end{array}$ & $\begin{array}{c}\log L_{2-10 \mathrm{keV}}^{\mathrm{obs}} \\
(7)\end{array}$ & $\begin{array}{c}\log L_{12 \mu \mathrm{m}} \\
\quad(8)\end{array}$ & $\begin{array}{l}\text { EW } \\
(9)\end{array}$ & $\begin{array}{c}+\mathrm{EW} \\
(10)\end{array}$ & $\begin{array}{c}-\mathrm{EW} \\
(11)\end{array}$ & $\begin{array}{l}\text { EW ref. } \\
\text { (12) }\end{array}$ \\
\hline 54 & AEGIS 602 & 0.769 & 214.8420 & 52.9219 & Bri14 & 42.2 & 44.5 & 2.58 & 0 & -1 & Boo18 \\
\hline 56 & XMMID 60152 & 0.579 & 150.3122 & 1.7302 & Lan 15 & 43.7 & 44.7 & 0.57 & 0.38 & 0.26 & Lan 15 \\
\hline 57 & CDFS 039 & 3.660 & 53.0785 & -27.8598 & Bri14 & 43.7 & 44.8 & 1.25 & 0 & -1 & Boo18 \\
\hline 58 & CDFS 454 & 0.650 & 53.0446 & -27.8019 & Bri14 & 41.0 & 44.8 & 5.00 & 0 & -1 & Boo18 \\
\hline 61 & COSMOS 0581 & 1.778 & 150.2910 & 2.0895 & Bri14 & 43.4 & 45.0 & 2.12 & 0 & -1 & Boo18 \\
\hline 62 & COSMOS 0987 & 0.353 & 149.7929 & 2.1256 & Bri14 & 42.0 & 45.1 & 1.54 & 0 & -1 & Boo18 \\
\hline 63 & $\begin{array}{c}\text { 2MASX } \\
\text { J03561995-6251391 }\end{array}$ & 0.108 & 59.0831 & -62.8609 & Ric15 & 43.4 & 45.1 & 0.20 & 0.19 & 0.03 & Boo18 \\
\hline 64 & CDFS 460 & 2.145 & 53.0976 & -27.7155 & Bri14 & 42.5 & 45.1 & 1.93 & 0 & -1 & Boo18 \\
\hline 65 & COSMOS 0363 & 2.704 & 150.0459 & 2.2013 & Bri14 & 44.4 & 45.2 & 0.91 & 0.96 & 0.60 & Boo18 \\
\hline 71 & IRAS F15307+3252 & 0.930 & 233.1838 & 32.7131 & H-L17 & 43.8 & 46.5 & 1.00 & 0.70 & 0.53 & Boo18 \\
\hline 72 & XMMID 324 & 1.222 & 53.2051 & -27.6806 & Geo13 & 43.5 & 46.6 & 0.51 & 0.91 & 0.15 & Geo13 \\
\hline
\end{tabular}

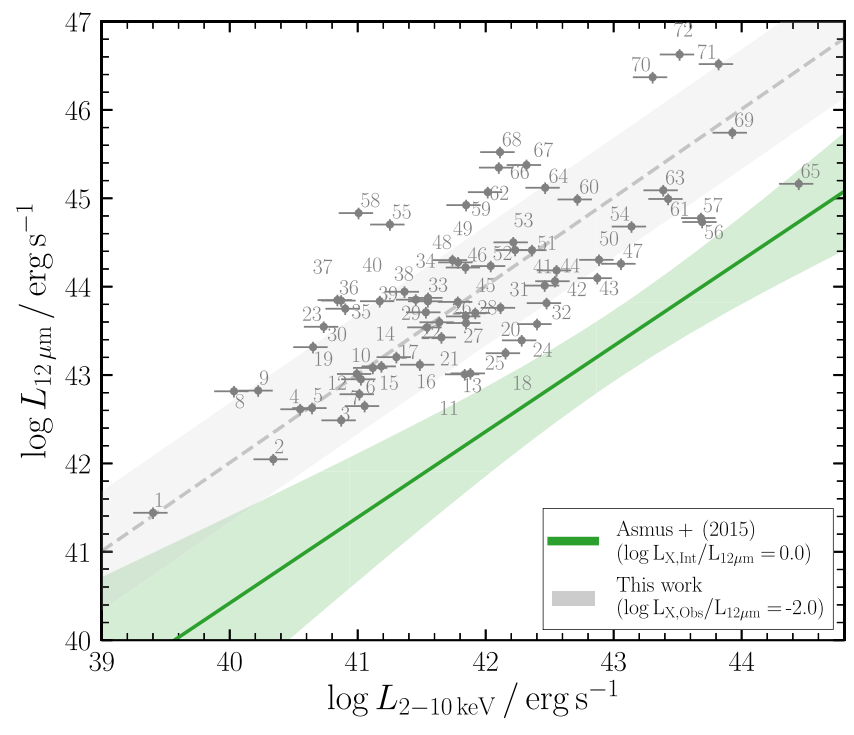

Figure 3. Predicted rest-frame $12 \mu \mathrm{m}$ source luminosity (interpolated from the Mullaney et al. (2011) infrared AGN spectral template) versus the restframe observed 2-10 keV luminosity of our sample of Compton-thick AGN. The solid (green) line is the best-fitting correlation from Asmus et al. (2015), together with the $1 \sigma$ scatter in light green shading. All sources were assigned a 30 per cent and 15 per cent uncertainty to the X-ray and $12 \mu \mathrm{m}$ luminosities, respectively. The sample shows diminished X-ray emission relative to $12 \mu \mathrm{m}$ emission by a factor of greater than two orders of magnitude on average, indicative of Compton-thick obscuration. The average observed correlation is shown with a grey dotted line and shaded standard deviation. Labels refer to the ID column in Table 1 .

sources with measured fluxes from Asmus et al., we use the quoted rest-frame $12 \mu \mathrm{m}$ luminosities and uncertainties therein.

\subsection{Rest-frame Fe K $\alpha$ line EWs}

Due to the complexity associated with NGC 1068 (Bauer et al. 2015), NGC 4945 (Puccetti et al. 2014) and the Circinus Galaxy
(Arévalo et al. 2014), our simplified phenomenological model could not provide a reasonable description of the data for these sources. For this reason, we use the EWs quoted in Ricci et al. (2015), converted to the rest-frame for the corresponding sources. Additionally, we did not have access to the spectral files for 4 high-redshift sources. The source of the EWs we use for our analysis are included in Table 1, column (12). In total, we computed the rest-frame neutral $\mathrm{Fe} \mathrm{K} \alpha$ fluorescence line EW for 65/72 sources, as follows:

(i) Any counts with $E<3 \mathrm{keV}$ in the source rest-frame were ignored for Chandra (or $E<4 \mathrm{keV}$ for NuSTAR) observations, in order to remove as much soft X-ray contamination from nonprimary AGN sources as possible. Such sources include intrinsic AGN emission scattered into the line of sight, a relativistic jet, $\mathrm{X}$-ray binaries present in the host or photoionized gas. Furthermore, all counts above $7 \mathrm{keV}$ in the observed frame were excluded to account for the instrument-based sensitivities of Chandra. The corresponding upper limit for NUSTAR was $\sim 14-15 \mathrm{keV}$ in the observed frame, optimizing the measurement of the continuum over the most sensitive NUSTAR energy range.

(ii) In the low counts regime, we used Cash-statistics (Cash 1979, c-stat) during fitting. Spectra were either grouped to allow a minimum number of counts, or a minimum signal-to-noise $(\mathrm{S} / \mathrm{N})$ ratio per bin, while retaining enough spectral resolution for the $\mathrm{Fe} \mathrm{K} \alpha$ line. We generally favoured fitting with C-stat unless sources had enough counts or high enough $\mathrm{S} / \mathrm{N}$ to warrant the use of $\chi^{2}$ statistics on a correspondingly S/N-binned spectrum. We experimented with different binning strategies within the sources fitted with C-stat, and found consistent outcomes.

(iii) Next we fitted each spectrum with a simplified phenomenological model consisting of photoelectric absorption acting on a composite power law plus a narrow Gaussian of FWHM $\approx 2 \mathrm{eV}$ $(\sigma=1 \mathrm{eV})$, modelling the observed continuum plus the narrow core of the Fe K $\alpha$ fluorescence line. This model was used only to constrain the shape of the observed spectrum, and the EW of the Fe K $\alpha$ line. If a given source had an observed excess of emission in the softer energy band ( $E \lesssim 4 \mathrm{keV}$ ) an apec component was additionally included in the model to account for this. In xspec, this 
baseline model takes the form:

$$
\begin{aligned}
\text { MODEL }= & \text { GAL_PHABS } \times(\text { APEC } \\
& + \text { ZPHABS } \times(\text { ZPOWERLAW }[\Gamma=1.4] \\
& \left.\left.+ \text { ZGAUSS }\left[E_{\mathrm{L}}=6.4 \mathrm{keV}\right]\right)\right) .
\end{aligned}
$$

GAL_PHABS refers to an additional minor contribution to the absorption from the Galaxy. Items in square brackets refer to fixed parameters. Although many studies suggest the intrinsic power law of AGN have average photon indices of $\sim 1.9$, we fit the spectra with a flatter (lower) photon index of 1.4 , as this is closer to the value found for the flat $(<10 \mathrm{keV})$ reflection spectra typically observed for Compton-thick AGN, and we required our model to provide a reasonable fit to the observed spectrum.

(iv) We then computed two-dimensional confidence contours over the ZPOWERLAW and ZGAUSSIAN model component normalizations (whilst leaving $N_{\mathrm{H}}$ and, if required to describe the soft region of the observed spectrum, the apec normalization, free).

(v) These contours were translated to confidence on the $\mathrm{Fe} \mathrm{K} \alpha$ $\mathrm{EW}$, and plotted as a function of the statistical test difference from the best-fitting acquired (chi-squared or Cash-statistics depending on the source). This enables us to determine the minimum, and hence presumed best-fitting rest-frame $\mathrm{EW}$, together with the $1 \sigma$ uncertainty. Irrespective of using chi-squared or Cash-statistic, we use a delta statistic of +2.30 to represent the $1 \sigma$ ( 68 per cent) confidence level for two interesting parameters. ${ }^{8}$

(vi) For sources in which the normalization of the $\mathrm{Fe} \mathrm{K} \alpha$ line could not be constrained in the fit, we use the limit derived by xspec on this parameter to calculate an upper bound on the EW. For any sources that yielded an unphysical EW $>5 \mathrm{keV}$, we set the limit to this value. This is applicable to three sources: CDFS 443, CDFS 454, and COSMOS 2180, with EW $\lesssim 12 \mathrm{keV}$, EW $48 \mathrm{keV}$, and $\mathrm{EW} \lesssim 11 \mathrm{keV}$, respectively. We defer the reader to the Appendix (Supplementary Information) for the grouped spectrum used for each source.

\subsection{Fitting procedure}

Our final sample consists of 72 sources, including 18 upper limits on the EW. All sources without quoted luminosities in Asmus et al. (2014) were assigned the same lower uncertainty of 0.3 dex on $12 \mu \mathrm{m}$ luminosity specified in Section 3.2. We then fitted a linear regression to the EW versus rest-frame $12 \mu \mathrm{m}$ luminosity. To account for all the uncertainties present in our sample whilst determining a fit, our fitting procedure was as follows: ${ }^{9}$

(i) The data set was bootstrapped by randomly sampling data points from the original whilst allowing repeats. The new data set was the same size as the parent sample.

(ii) Each point in the bootstrapped data set was randomly resampled depending on the uncertainty of each point, as follows:

(a) Non-detections/upper limits: New points were randomly drawn from a uniform distribution in the interval [ $\log 100 \mathrm{eV}, \log$ limit [eV]].

(b) Detections: A new value was generated from a Gaussian distribution with standard deviation given by the $1 \sigma$ error being considered for that point.

\footnotetext{
${ }^{8}$ https://heasarc.gsfc.nasa.gov/xanadu/xspec/manual/XSappendixStatistics .html

${ }^{9}$ Similar in method to Bianchi et al. (2007).
}

To avoid strongly unphysical values from biasing the simulations, we truncated the randomized EWs to between $100 \mathrm{eV}$ and $5 \mathrm{keV}$.

(iii) A linear least-squares regression was carried out on the Monte Carlo simulated data set using the scipy. linregress PYTHON package. The Spearman's Rank correlation coefficient $(\rho)$ was then found using the scipy.spearmanr package for each fit.

(iv) Steps (i)-(iii) were repeated in order to obtain a distribution of gradients, $y$-intercepts and $\rho$ values for the original data set.

\section{RESULTS}

Table 1 includes details of each source used in our final sample, and the Appendix (Supplementary Information) contains the bestfitting spectrum and EW contour for each source used, as well as the sources ruled out in our analysis. After carrying out 20000 iterations, we obtain a best-fitting linear regression to the data of

$$
\begin{aligned}
& \log \left(\mathrm{EW}_{\mathrm{Fe} \mathrm{K} \alpha} / \mathrm{keV}\right) \\
& \quad=-(0.08 \pm 0.04) \log \left(L_{12 \mu \mathrm{m}} / 10^{44} \mathrm{erg} \mathrm{s}^{-1}\right)+2.87 \pm 0.05
\end{aligned}
$$

Fig. 4 shows all rest-frame $12 \mu \mathrm{m}$ luminosities versus rest-frame neutral $\mathrm{Fe} \mathrm{K} \alpha$ fluorescence line EWs. Blue arrows represent upper limits. As a comparison to previous studies into the IwasawaTaniguchi effect, we further include the gradients of previous works: Iwasawa \& Taniguchi (1993), Page et al. (2004), Bianchi et al. (2007), and Ricci et al. (2014), normalized to the same $y$-intercept at $10^{44} \mathrm{erg} \mathrm{s}^{-1}$. We make this normalization since the EWs we report for our sample are measured relative to the observed spectrum, which for Compton-thick obscuration is drastically different to the observed spectrum for unobscured AGN, not to mention our proxy for the bolometric luminosity is different to that previously used by other studies.

To test the significance of the fit, we computed the Spearman's Rank correlation coefficient $(\rho)$ of the correlation for our sample, excluding upper limits. Upper limits were excluded since $\rho$ tests the strength of a monotonic relationship between variables, which can be dramatically affected by the large range of values/orders of variables attainable with the inclusion of limits in our Monte Carlobased fitting method. This left 54 sources, and gave a value of $\rho=-$ $0.28 \pm 0.12$. Fig. 5 shows the corresponding distribution in $\rho$ found, indicating a negative correlation to 98.7 per cent confidence.

Our best-fitting gradient is fully consistent with Ricci et al. (2014) within $1 \sigma$ errors, who attempted to take into account time-averaging of the spectra for determining EWs - see Section 5 for further discussion on this result. The gradient found here is also flatter than the Bianchi et al. (2007) best-fitting gradient, but consistent within 90 percent confidence. We include the distributions of our best linear fit gradients and $y$-intercepts in Figs 6(a) and (b), respectively, for the 20000 iterations (including upper limits).

\section{DISCUSSION}

Our results indicate the presence of an Iwasawa-Taniguchi effect for Compton-thick AGN. This is surprising, since the majority of our sample is presumed to have a noticeable flux contribution from the reflected component in the $\sim 6-7.9 \mathrm{keV}$ energy region (e.g. Fig. $1-$ we will address this further in Section 5.2.1), and the Fe K $\alpha \mathrm{EW}$ is not expected to vary relative to the underlying Compton-scattered reflection continuum. Here, we discuss the significance of our result, 
Table 2. Summary of the anticorrelation probability significances found for the studies into the Iwasawa-Taniguchi effect included in Fig. 4. The table lists the (1) study reference, (2) the correlation metric used to quantify the significance of the resulting anticorrelation: 'Prob.' - probability of an anticorrelation; ' $\rho$ ' - Spearman's Rank correlation coefficient; 'Corr. coeff.' - correlation coefficient, (3) the value of the correlation metric found by the work, (4) the number of objects in each sample, and (5) the obscuration classes of the sources included in the corresponding work.

\begin{tabular}{lcccc}
\hline Reference & Metric & Value & No. of sources & Obscuration class \\
& $(1)$ & $(3)$ & 54 & (4) \\
\hline This work & Prob. & 98.7 per cent & 54 & Compton-thick \\
Ricci et al. (2014) & Prob. & 99 per cent & 47 & Seyfert 2 \\
Bianchi et al. (2007) & Prob. & 99.6 per cent & 157 & Unobscured \\
Jiang et al. (2006) & $\rho$ & -0.47 & 101 & Unobscured \\
Page et al. (2004) & Prob. & $>99.98$ per cent & 53 & Unobscured \\
Iwasawa \& Taniguchi (1993) & Corr. coeff. & $>0.8$ & 37 & Unobscured \\
\hline
\end{tabular}

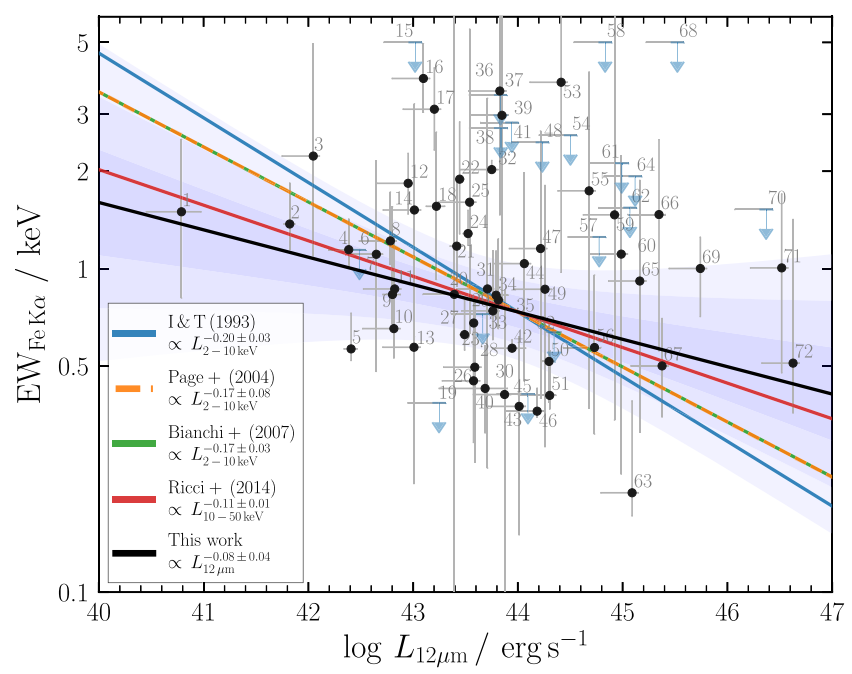

Figure 4. Plot of $L_{12 \mu \mathrm{m}}$ versus (rest-frame) neutral $\mathrm{Fe} \mathrm{K} \alpha$ fluorescence line EWs. Blue arrows represent upper limits. As a comparison to previous studies into the Iwasawa-Taniguchi effect, we further include the gradients of previous works Iwasawa \& Taniguchi (1993, I\& T (1993)), Page et al. (2004, Page+(2004)), Bianchi et al. (2007, Bianchi+(2007)), and Ricci et al. (2014, Ricci+ (2014)). These correlations have all been renormalized to match our best-fitting $y$-intercept at $10^{44} \mathrm{erg} \mathrm{s}^{-1}$ for comparison, since we are using the $12 \mu \mathrm{m}$ luminosity, which is different from the intrinsic luminosity proxies used by other Iwasawa-Taniguchi effect studies. The blue shaded region represents the standard deviation from the mean of our best fit, with lighter shading corresponding to incrementally lower numbers of standard deviation.

as well as possible physical interpretations if confirmed on larger samples.

\subsection{Significance of result}

As a comparison to previous works, Table 2 includes the anticorrelation significance metric quoted for the studies into the IwasawaTaniguchi effect that we include in Fig. 4, as well as the obscuration type of the sources included in the corresponding samples.

\subsubsection{AGN dominance}

In addition to estimating the effect of star formation contamination using high-angular-resolution observations (see Section 3.2.1), we sought to test the AGN dominance of our interpolated infrared luminosities. We use the colour criteria of Stern et al. (2012), Mateos et al. (2012), and Lacy et al. (2007) for observed flux densi-

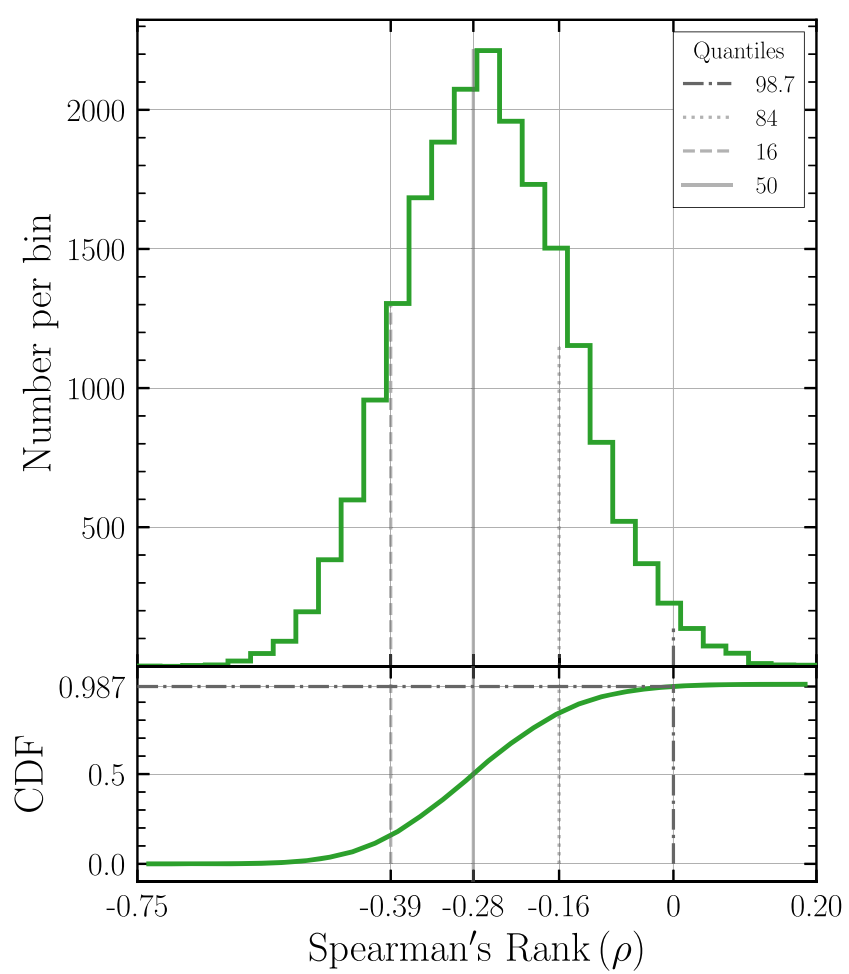

Figure 5. Distribution of Spearman's Rank correlation coefficients $(\rho)$ generated from 20000 iterations of steps (1)-(3) of our fitting procedure, outlined in Section 3.4, for a data set excluding all upper limits (54 in total). The 16th, 50th, and 84th quantiles are shown with dashed, solid, and dotted lines, respectively. The dotted (purple) line additionally shows the correlation to be negative to 98.7 per cent confidence.

ties (used to renormalize the Mullaney et al. 2011 AGN template and interpolate a rest-frame $12 \mu \mathrm{m}$ flux) from WISE and Spitzer, respectively. However, Stern et al. (2012) do note that the efficiency of such mid-infrared colour selections of AGN increases strongly with X-ray luminosity. As such, lower luminosity sources, e.g. with $L_{2-10 \mathrm{keV}} \lesssim 10^{43} \mathrm{erg} \mathrm{s}^{-1}$, in our sample may not display AGN-like mid-infrared colours, and thus lie outside the wedge and cut thresholds. Alternatively, the sources lying outside the selection criteria may not be intrinsically weak - for example, Gandhi et al. (2015a) notes that bluer $W 1-W 2$ colours could arise from strong host star formation contamination or anisotropic/weak reprocessed torus emission. As such, sources satisfying any one of these three colour thresholds are most likely to not display star formation contamination, and be AGN-dominated in the mid-infrared. The flux densities are plotted in Fig. 7. In total, 38/72 sources satisfy ei- 

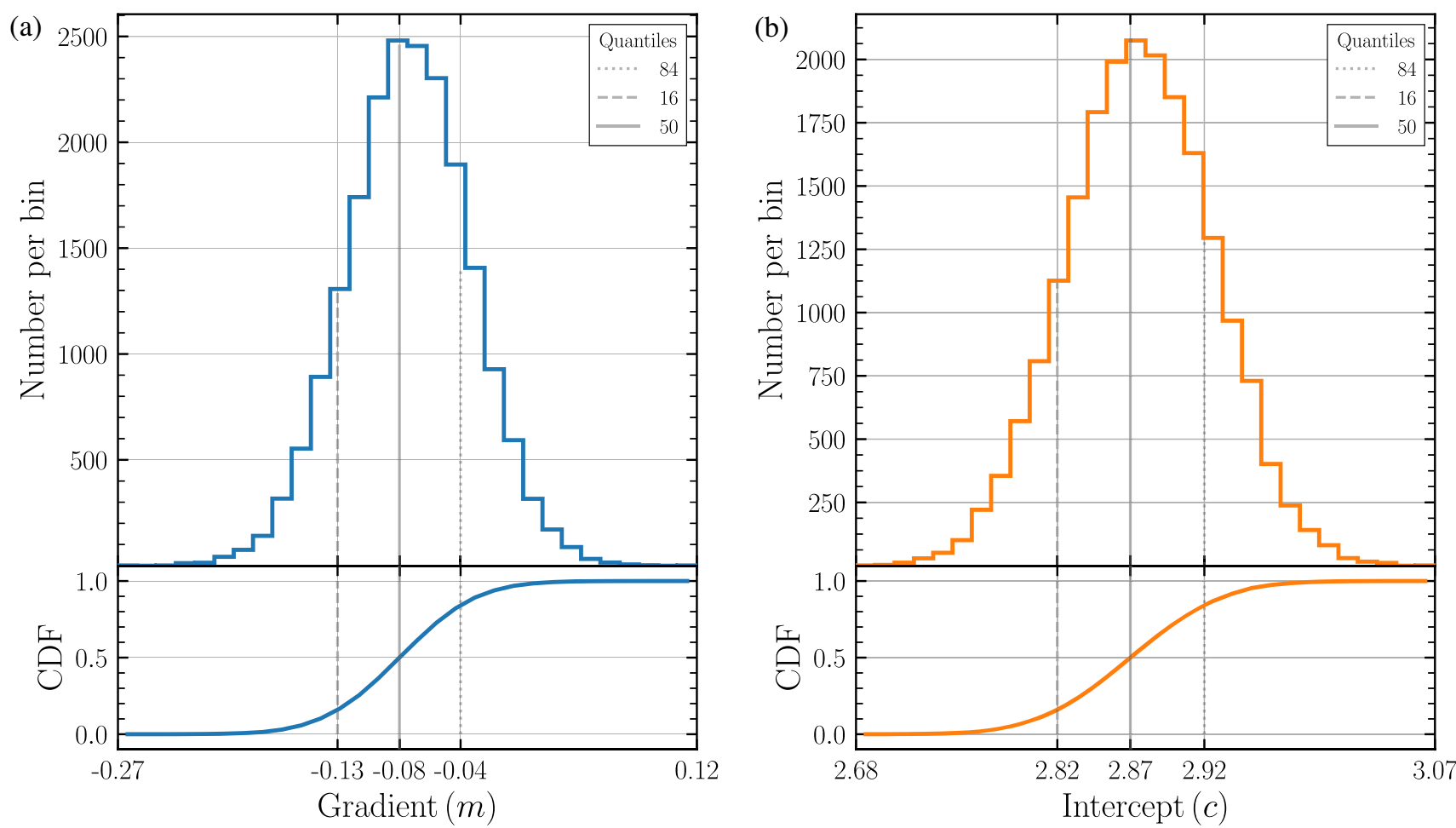

Figure 6. (a) Distribution of linear regression gradients generated from 20000 iterations of steps (1)-(3) of our fitting procedure (Section 3.4). The 16th, 50th, and 84th quantiles are shown with dashed, solid, and dotted lines, respectively. (b) Distribution of linear regression $y$-intercepts generated from 20000 iterations of steps (1)-(3) of our fitting procedure, outlined in Section 3.4. The 16th, 50th, and 84th quantiles are shown with the same line styles as in figure (a).

ther Stern et al. (2012) and Mateos et al. (2012) combined (for WISE-based observations) or Lacy et al. (2007) (for Spitzer-based observations). This potentially indicates some form of star formation contamination (or another form of contaminant) present in the sources that do not satisfy these criteria. However, running a fit to only the 38 predicted AGN-dominated sources in our sample results in a gradient of $m=-0.04 \pm 0.06$ between EW and $L_{12 \mu \mathrm{m}}$, fully consistent with the main fit presented in Section 4, albeit with larger uncertainty.

As a further test of contamination in our sample, we carried out a fit only to sources displaying a deficit in observed X-ray to $12 \mu \mathrm{m}$ luminosity of greater than two orders of magnitude (see Fig. 3). This again returned a consistent result with our main fit of $m=-0.08 \pm 0.05$ for 36 sources. These tests suggest that any star formation contamination does not dominate the trend that we observe.

\subsubsection{Binning}

Lastly, we carried out a fit to the sample binned by $12 \mu \mathrm{m}$ luminosity. A maximum binning of nine sources optimized total number of bins together with sources per bin. We approximated all upper limits as the average between the limit and $\log 100 \mathrm{eV}$, then assigned a $1 \sigma$ error to the new point that encompassed $\log 100 \mathrm{eV}$ to the limit. The corresponding binned EW error for each bin was then given by

$\sigma_{\mathrm{EW}}=\frac{\sqrt{\sum \delta \mathrm{EW}^{2}}}{N}$.

Here, $N$ refers to the number of sources in each bin. The best-fitting gradient we get to the binned data is $\log \left(\mathrm{EW}_{\mathrm{Fe} \mathrm{K} \alpha}\right) \propto$
$-0.06_{-0.08}^{+0.05} \log \left(L_{12 \mu \mathrm{m}}\right)$, and is plotted in Fig. 8 with a red solid line and one standard deviation shading. The best fit to the original sample is shown with a dashed (black) line, which is fully consistent with the binned gradient. The binned data has been renormalized to have the same $y$-intercept as the original result for easier visual comparison of gradients. The background grey points show the original data.

\subsection{Physical interpretation}

\subsubsection{Covering factor dependence}

The EW of the $\mathrm{Fe} \mathrm{K} \alpha$ line measured solely relative to the reprocessed continuum is very rarely $<1 \mathrm{keV}$ (see fig. 8 of Murphy \& Yaqoob 2009). One way to achieve an observed EW significantly less than $1 \mathrm{keV}$ is via leaked intrinsic AGN emission contributing some flux to the observed spectrum in the iron line region. An example of this would be in transmission-dominated Compton-thick AGN, or a 'clumpy' torus. Typical X-ray spectral model predictions (see Fig. 1) for AGN show that for column densities, $N_{\mathrm{H}} \gtrsim$ $1.5 \times 10^{24} \mathrm{~cm}^{-2}$, the observed reflected flux is $\gtrsim 10$ times more than the transmitted flux. Below this column density, a borderline Compton-thin/thick AGN could have a reasonable contribution in flux from the transmitted component, and hence a variable continuum with intrinsic luminosity. One way to obtain less reflected flux with increasing column would be a decreased covering factor, which has been dubbed the 'receding torus', and was suggested to explain the Iwasawa-Taniguchi effect for unobscured and mildly obscured sources previously (e.g. Page et al. 2004; Ricci et al. 2013b, see Section 1 of this paper). However, Lawrence \& Elvis (2010) discuss 

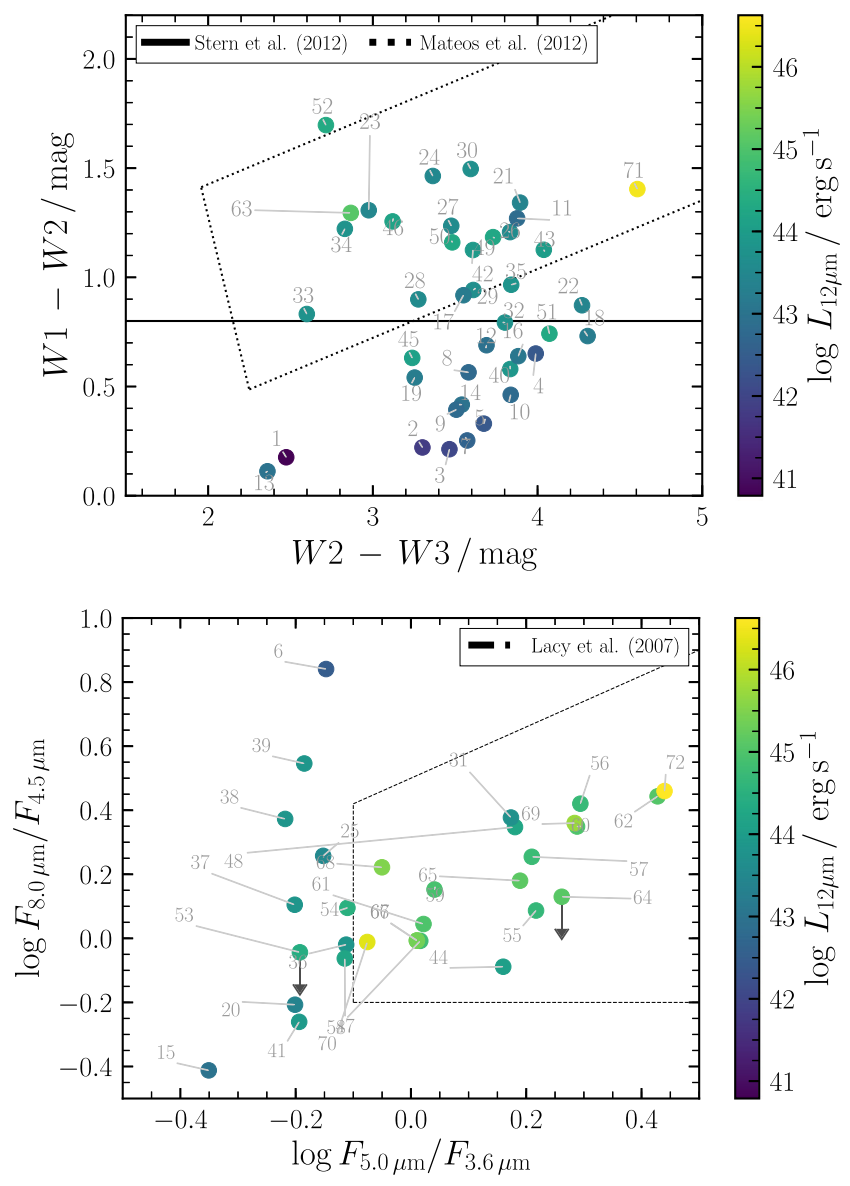

Figure 7. Colour selection criteria for WISE (top panel) and Spitzer (bottom panel) flux densities. The WISE thresholds are from Stern et al. (2012) and Mateos et al. (2012), and the Spitzer colour wedge is from Lacy et al. (2007). In total, 38/72 sources lie outside these criteria, but after testing the possible effects of star formation on these sources, we still require a significant anticorrelation to fit the data - see Section 3.2.1 for details.

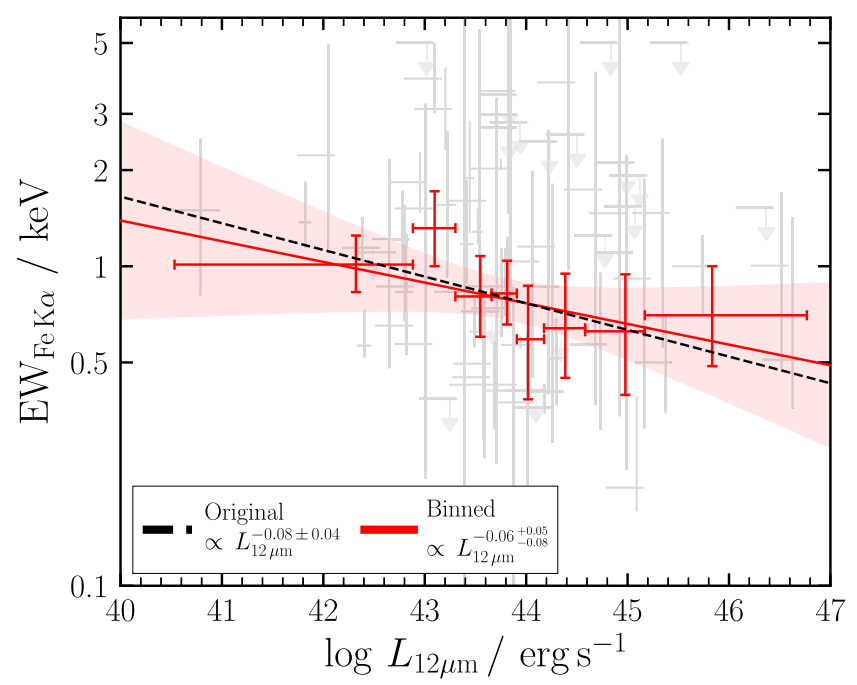

Figure 8. The best fit to the binned sample, with a maximum of nine sources per bin (see the text for details of the binning method used). The red shaded region shows the standard deviation from the mean of our fit, which is fully consistent with the original best fit found for the unbinned data (shown with a black dotted line). This has been renormalized to the same $y$-intercept as the binned fit to allow an easier comparison between gradients. Background grey points show the source data that was binned.

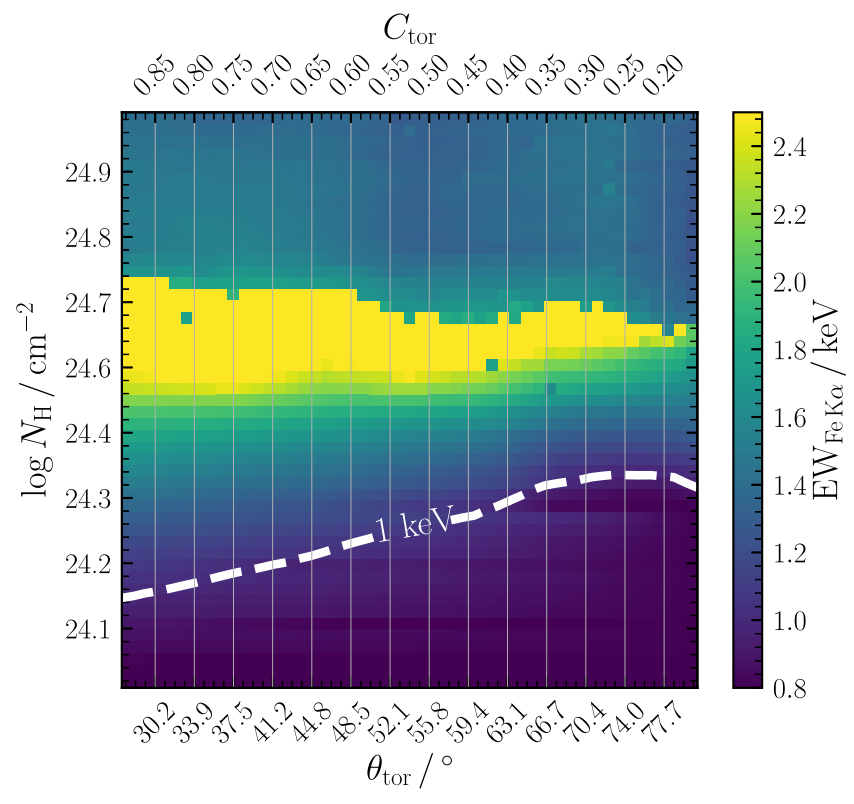

Figure 9. A colour map of simulated neutral iron $\mathrm{K} \alpha \mathrm{EW}$ for a series of lineof-sight column densities $\left(N_{\mathrm{H}}\right)$ and torus covering factors $\left(C_{\text {tor }}=\cos \theta_{\text {tor }}\right)$ in the borus 02 (Baloković et al. 2018) model. For these simulations, the line-of-sight column density and equatorial column density were tied together. Spectra were simulated using the NUSTAR response matrices. The simulated spectra were then fit in the 6-7 keV energy region with a POWERLAW + GAUSSIAN to derive a predicted EW. The contour shows the boundary at which all EW are predicted to be $>1 \mathrm{keV}$. All EWs predicted to be $<0.8$ or $>2.5 \mathrm{keV}$ were capped (shown with the same minimum and maximum colour, respectively). Due to our selection of sources with $\log N_{\mathrm{H}}>24.18$ $\left(N_{\mathrm{H}}>1.5 \times 10^{24} \mathrm{~cm}^{-2}\right)$ to 90 per cent confidence from literature values, we cannot rule out sources as having at least a partial EW dependence with covering factor, as can be seen by this region of the colour map.

that the apparent decrease of obscured AGN fraction with bolometric luminosity is much less significant in IR/radio samples than with $\mathrm{X}$-ray samples, suggesting that the receding torus model may not exist in nature.

In Fig. 9, we show a colour map of simulated EWs predicted from the borus 02 model for an $\sim$ edge on $\left(84^{\circ}\right)$ viewing angle, with varying column densities and covering factors. All spectra were simulated using fakeit from within xspec with the $N u S$ TAR simulation files provided by the NUSTAR team, ${ }^{10}$ and then the resulting spectrum was re-fit in the $6-7 \mathrm{keV}$ energy region by a POWERLAW + GAUSSIAN model. The EW of a narrow (FWHM $\sim 2 \mathrm{eV})$ Gaussian was then derived at fixed line centroid $\left(E_{\mathrm{L}}=6.4 \mathrm{keV}\right.$, using the eqwidth command in xspec. We also overplot the limiting contour at which all EWs are $>1 \mathrm{keV}$, which we take as a proxy for reflection dominance. Interestingly, for lower covering factors (higher opening angles), the column density can be high $\left(\log \mathrm{N}_{\mathrm{H}}>24.3\right.$ in some cases), and still feature a spectrum with presumably leaked transmitted emission. Since our original $N_{\mathrm{H}}$ selection was $\log N_{\mathrm{H}}>24.18\left(N_{\mathrm{H}}>1.5 \times 10^{24} \mathrm{~cm}^{-2}\right)$ to 90 per cent confidence from literature values, from this plot we cannot rule out that the higher luminosity sources (with assumed lower covering factors) would feature some sort of leaked transmitted flux contributing to the continuum around $6.4 \mathrm{keV}$ and decreasing observed neutral $\mathrm{Fe} \mathrm{K} \alpha \mathrm{EW}$. Furthermore, if the luminosity-dependent cov-

${ }^{10} \mathrm{https}: / /$ www.nustar.caltech.edu/page/response_files 
ering factor explanation is correct, then we are currently lacking such reflection-dominated Compton-thick AGN at high luminosities since the $\mathrm{Fe} \mathrm{K} \alpha \mathrm{EW}$ is predicted to always be greater than $1 \mathrm{keV}$ for $N_{\mathrm{H}} \gtrsim 1.5 \times 10^{24} \mathrm{~cm}^{-2}$.

As an additional test, we selected sources with literature best fit lower 90 percent uncertainty on the column density to be $N_{\mathrm{H}}>1.5 \times 10^{24} \mathrm{~cm}^{-2}$. This returned 14 sources, with only two sources at higher redshift (COSMOS 0363; $z=2.704$ and BzK $4892 ; z=2.578$ ). Although the corresponding best fit to this sample returns a gradient of $m=-0.10_{-0.08}^{+0.07}$, which is entirely consistent with the result for the full sample, we lack enough robustly reflection-dominated sources at higher redshifts to draw precise conclusions for a transmitted component altering the narrow $\mathrm{Fe} \mathrm{K} \alpha$ line.

An alternative way to detect a considerable contribution from the transmitted component in Compton-thick AGN would be via changing-look AGN variability. In this scenario, the Compton-thick obscurer is clumpy, enabling clouds of differing column to traverse the line of sight, potentially resulting in leaked intrinsic emission. Such extreme eclipsing events from Compton-thin to Comptonthick levels of obscuration have been observed previously (Risaliti et al. 2007), but are rare. As such, this is unlikely to be responsible for the diminished $\mathrm{Fe} \mathrm{K} \alpha$ lines observed in all higher luminosity sources of the sample where we see the greatest decrease in EW, but may play a non-negligible role.

\subsubsection{Ionization}

Finally, we consider the effects of ionization on Compton-thick Xray spectra. As mentioned in Section 1, many obscured candidate AGN not only show diminished neutral $\mathrm{Fe} \mathrm{K} \alpha$ line EWs, but also increased ionized $\mathrm{Fe} \mathrm{K} \alpha$ EWs with intrinsic luminosity (Iwasawa et al. 2009). Indeed, a correlation between spectral slope (a proxy for the accretion efficiency of AGN) with the $\mathrm{Fe} \mathrm{K} \alpha$ line energy was found by Dewangan (2002). From Fig. 4, one would expect this effect to be most prevalent for intrinsically bright (i.e. high $L_{\text {bol }}$ and/or high Eddington ratio) systems, which may be more intrinsically UV-luminous relative to X-rays.

To robustly test this would require an ionized toroidal X-ray reprocessing model, which is currently unavailable. For this reason, we use the xillver (García et al. 2013) disc reflection model, which calculates a spectrum from the accretion disc surrounding AGN including reflection and also ionized emission lines. Fig. 10 illustrates the approximate EWs of the ionized 6.70 and $6.97 \mathrm{keV}$ iron emission lines and neutral $\mathrm{Fe} \mathrm{K} \alpha$ line as a function of ionization parameter. This is defined as $\xi=4 \pi F_{x} / n_{e}$ (García et al. 2013), where $F_{x}$ is the net flux in the 1-1000 Ry energy region, and $n_{e}$ is the electron number density. From Fig. 10, one can infer that the dominance of ionized lines increases with respect to the neutral ones for high values of $\xi$. Thus, ionization could be a potential explanation for our results.

\subsubsection{Other possibilities}

Another possibility for a depleted neutral iron line in a Comptonthick candidate AGN would be dilution of the reflection spectrum by scattered primary emission from the AGN that is reprocessed by a diffuse ionized 'mirror' in a line-of-sight direction of lower column density. Such a component would scale with intrinsic luminosity and thus contribute to the Iwasawa-Taniguchi effect. In fact, a considerable scattered fraction of intrinsic emission was found

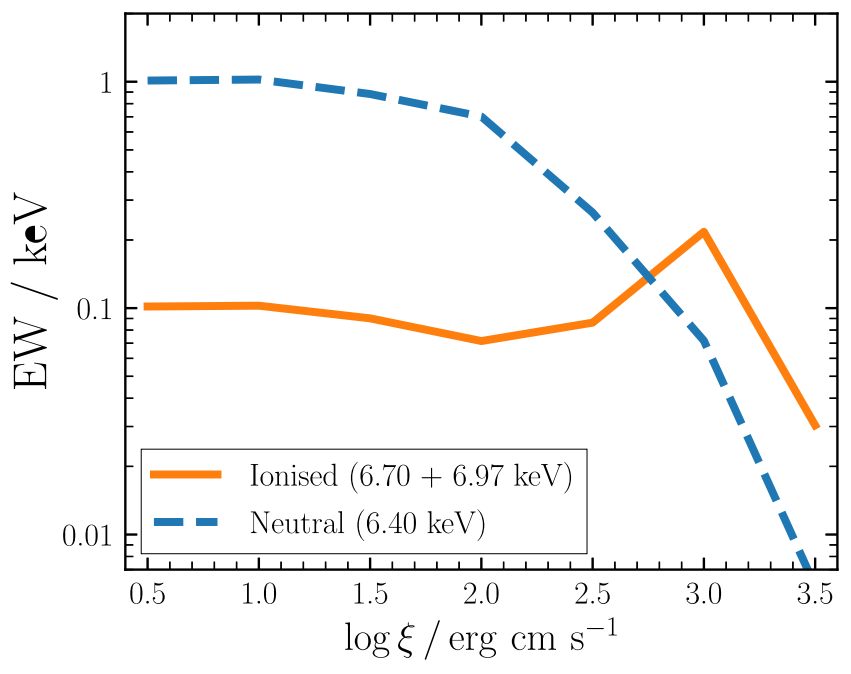

Figure 10. Xillver simulated EWs of the ionized iron lines at 6.70 and $6.97 \mathrm{keV}$ (solid orange line) together with neutral $\mathrm{Fe} \mathrm{K} \alpha$ line $\mathrm{EW}$ (dotted blue line) as a function of ionization parameter. The EWs were calculated by integrating the flux in the continuum between 5.9 and $7.2 \mathrm{keV}$, subtracted from the flux in the lines (integrated $\pm 0.05 \mathrm{keV}$ of each predicted line centroid), and then divided by the interpolated continuum at the line centroid. The spectra were simulated using the xillver spectral model, assuming an inclination of $18.2^{\circ}$ ( face-on, i.e. maximum reflection).

to explain the observed X-ray spectrum of the local Compton-thick AGN NGC 7674 by Gandhi et al. (2017). The authors find a fraction of $\sim 2-10$ per cent to 90 per cent confidence could explain the low observed EW of the neutral $\mathrm{Fe} \mathrm{K} \alpha$ line from this Compton-thick source.

A second tentative explanation for an obscured IwasawaTaniguchi effect comes from dual AGN candidate systems, in which a spatially unresolved, less-obscured AGN is present in combination with a heavily obscured source. Supermassive black hole evolution simulations (from, e.g. Hopkins et al. 2008) predict luminous quasars to originate from gas-rich mergers. Immediately post-merger, these sources are predicted to be deeply embedded in the large dust and gas reservoirs that are rapidly being accreted, which absorb optical to X-ray emission and reprocess this at infrared wavelengths. Depending on the spatial separation of the merging supermassive black holes, the extracted X-ray spectrum could actually be the combined contribution from two components of differing obscuration levels. Koss et al. (2016) used NuSTAR to spatially resolve the emission from the dual AGN in NGC 6921. The authors found the two AGN components to be Compton-thick, but were able to separately study each independently in the $>10 \mathrm{keV}$ waveband. If one component of a dual AGN were less obscured, but contributed a considerable proportion of the total flux contribution, this could result in a diminished $\mathrm{Fe} \mathrm{K} \alpha$ complex in some postmerger candidates such as hyperluminous $\left(L_{8-1000 \mu \mathrm{m}}>10^{13} \mathrm{~L}_{\odot}\right)$ infrared galaxies, Dust Obscured Galaxies (Dey et al. 2008, DOGs) and Hot DOGs (Wu et al. 2012). For example, recent works have postulated the presence of dual AGN in NGC 7674 (Kharb, Lal $\&$ Merritt 2017) and Mrk 273 (Iwasawa et al. 2018). Furthermore, Vito et al. (2018) recently studied the X-ray emission from $20 \mathrm{Hot}$ DOGs, and found typical predicted X-ray line-of-sight column densities of $N_{\mathrm{H}} \sim 1-1.5 \times 10^{24} \mathrm{~cm}^{-2}$. This is illustrated in Fig. 11, in which we plot the composite spectrum in black (solid line), formed by combining the spectrum from a reflection-dominated AGN $\left(\log N_{\mathrm{H}} / \mathrm{cm}^{-2}=24.5\right.$; red dashed line) and an unobscured 


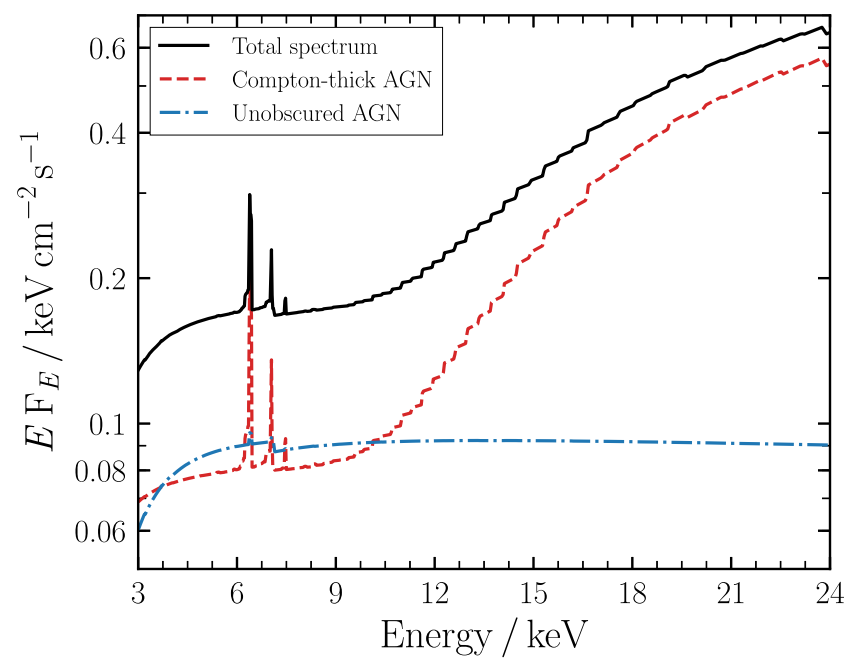

Figure 11. Dual-AGN schematic to explain the lower observed Fe K $\alpha$ EWs observed in luminous and ultraluminous infrared galaxies $\left(L_{8-1000 \mu \mathrm{m}}>\right.$ $10^{11} \mathrm{~L}_{\odot}$ and $L_{8-1000 \mu \mathrm{m}}>10^{12} \mathrm{~L}_{\odot}$, respectively). If an AGN were observed post-merger, the two supermassive black holes could exist temporarily separately, but spatially unresolved, leading to an observed (solid black line) composite spectra from two AGN. The individual unobscured (dotdashed, blue) and obscured (dashed, red) predicted AGN spectra are also plotted. Depending on the relative contributions in observed flux from either component, the total spectrum could have characteristics of a mildly obscured AGN, despite containing a heavily obscured source. Both the AGN spectra were simulated using the borus 02 model.

AGN $\left(\log N_{\mathrm{H}} / \mathrm{cm}^{-2}=22\right.$; blue dot-dashed line). Higher angular resolution X-ray instruments would be required to separate the two components and stringently test this hypothesis. We further note that both a strong scattered component as well as dual AGN would struggle to explain the observed prominence of ionized iron emission lines often observed in the X-ray spectra of infrared-luminous systems (Gilli et al. 2014; Teng et al. 2014; Farrah et al. 2016).

A final possibility arises from the effects of dust grains on Xray photons. Typical X-ray reprocessing spectral models consist of ray-tracing through a dust-free gas, but not all consider the possible effects that dust grains have on the observed X-ray spectrum in detail. In fact, Draine (2003) has shown that $\sim 90$ per cent of the incident power at energy $E=6.4 \mathrm{keV}$ on dust grains scatter with angle, $\theta_{\mathrm{s}}<0.05^{\circ}$, relative to the incident photon direction. Gohil $\&$ Ballantyne (2015) further found that such a large anisotropic emission associated with dust grains, as opposed to the isotropic emission of hot gas typically invoked in X-ray reprocessing torus models could enhance the Fe $\mathrm{K} \alpha$ line EW relative to the underlying reflection continuum by up to factors of $\sim 8$ for Compton-thick gas. More luminous AGN would be expected to have a larger dust sublimation radius, and thus have an altered $\mathrm{Fe} \mathrm{K} \alpha \mathrm{EW}$ relative to the less luminous sources, presumably with smaller dust sublimation radii. Such a scenario effectively decouples the $\mathrm{Fe} \mathrm{K} \alpha$ line from the underlying reflection continuum, and there is already tentative evidence suggesting that these two components may arise from physically separate regions within the torus dust sublimation zone (Gandhi, Hönig \& Kishimoto 2015b).

\subsection{Implications}

\subsubsection{Redshift evolution of Compton-thick AGN}

As stated earlier, multiple works predict the obscured fraction of AGN to decrease with increasing luminosity and/or Eddington fraction (Ueda et al. 2011; Merloni et al. 2014; Georgakakis et al. 2017). In addition, since the number of luminous AGN is predicted to increase with redshift, this would imply a redshift evolution of obscuration amongst AGN. However, the anticorrelation we report could lead to a correction to X-ray inferred column densities, that were derived based on fitting an observed iron line. From Fig. 4, this correction factor would be largest for the most luminous sources. Depending on the relative contributions at different luminosities, this could then alter the obscured fraction dependence with luminosity. Some evidence has indicated a weak or no evolution of the obscured AGN fraction. For example, Vito et al. (2014) studied a sample of $141 \mathrm{X}$-ray selected AGN at $3<z \leq 5$ and found no evidence for an anticorrelation between obscured fraction and luminosity, despite suggesting that this may be due to the non-detection of the lower luminosity obscured sources at higher redshift. In contrast, Mateos et al. (2017) only found a weak luminosity dependence of the type 2 AGN fraction for covering factors derived from infrared clumpy torus modelling for $z \leq 1$, and Buchner et al. (2015) further found a constant Compton-thick fraction with redshift or accretion luminosity for a sample of $\sim 2000$ AGN.

To test if our result is biased by redshift, we further separated our sample into two redshift bins, below and above the median redshift of 0.038 . Fig. 12 shows the redshift distribution of our sample with the median redshift shown with an orange (vertical) line. Carrying out a fit to either redshift bin independently yielded consistent gradients of $m=-0.19_{-0.07}^{+0.06}$ and $m=-0.04 \pm 0.08$, for the low- and high-redshift bins, respectively, albeit with large scatter.

\subsubsection{The growth rate of $A G N$}

Current X-ray reprocessing torus models do not account for the possible effects of dust grains, and/or reflector ionization on the observed reflection spectrum. To zeroth order, such a model could interpret a less prominent neutral $\mathrm{Fe} \mathrm{K} \alpha$ line as evidence for a lower obscuring column than the true value for intrinsically bright, heavily obscured objects if our results are confirmed using larger sample studies. In the most extreme case, a Compton-thick system could be predicted to be only mildly obscured. From Fig. 3, this would mean underpredicting the intrinsic X-ray luminosity, and hence the growth rate of such systems, potentially by factors of around two orders of magnitude.

\subsection{The future}

Clearly one of the major sources of uncertainty in the relation we report here is on the $\mathrm{EW}$ of the $\mathrm{Fe} \mathrm{K} \alpha$ line. However, many future $\mathrm{X}$-ray missions can improve on this uncertainty, such as the $X$-ray Astronomy Recovery Mission (XARM) ${ }^{11}$ or Athena (Nandra et al. 2013). $X A R M$ will enable high spectral resolution studies of the iron line (e.g. Hitomi Collaboration et al. 2017) and be able to test the ionization scenario directly. Additionally, Athena will probe high-redshift Compton-thick AGN sensitively. Fig. 13 shows the possibilities with the Athena Wide Field Imager (WFI), with a $20 \mathrm{ks}$ simulated spectrum shown in purple together with the original $4 \mathrm{Ms}$

${ }^{11}$ https://heasarc.gsfc.nasa.gov/docs/xarm/ 


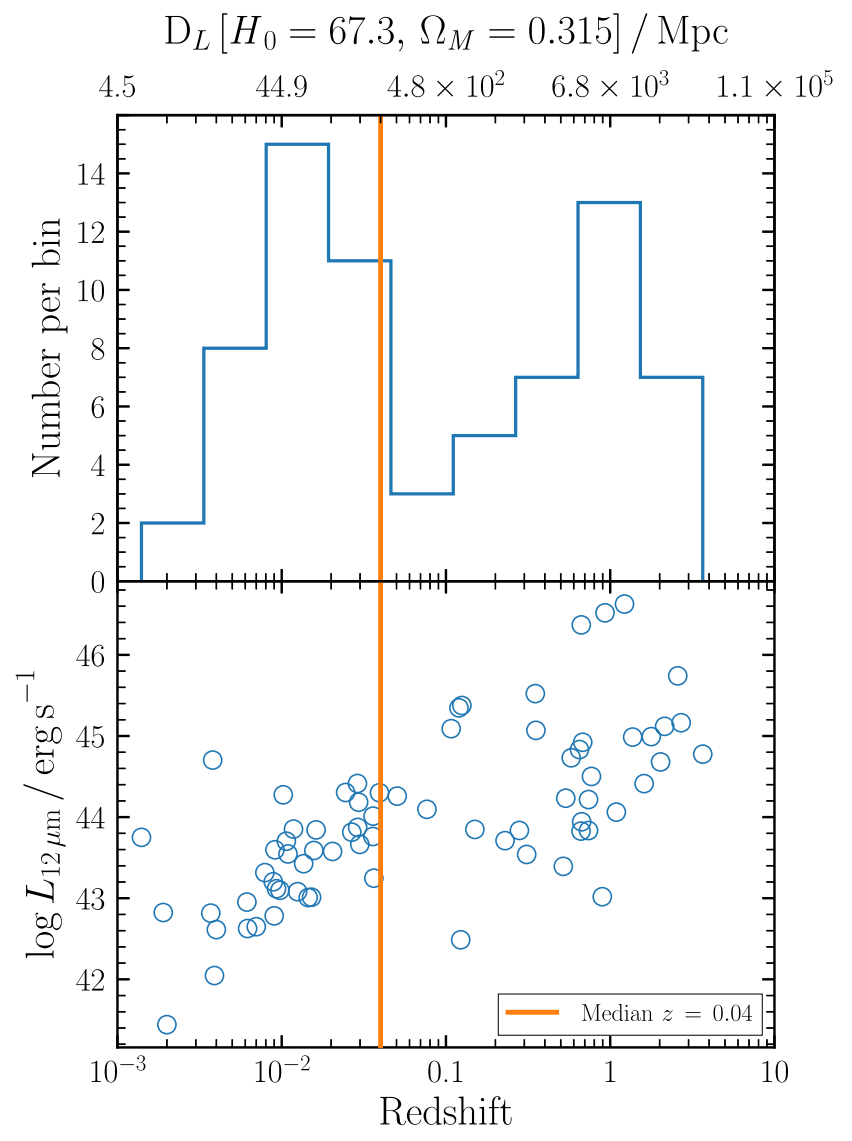

Figure 12. Redshift distribution of our sample, according to the rest-frame $12 \mu \mathrm{m}$ luminosity. The median redshift was chosen to split the sample into two redshift bins, in order to provide comparable numbers of sources in either bin.

CDFS observed spectrum for CDFS 384 from our sample, shown in black. Clearly the signal to noise is dramatically enhanced at the $\mathrm{Fe} \mathrm{K} \alpha$ (rest-frame $6.4 \mathrm{keV}$ ) line as well as the neighbouring continuum, enabling a huge improvement on the calculated EW contour in the lower panel of the figure. The simulated spectrum was calculated from the original best-fitting model to the observed Chandra spectrum. What this figure clearly shows, however, is that the confidence range on the EW of such obscured objects will be powerfully improved with the advent of such high-sensitivity instruments in the future.

\section{SUMMARY}

Here, we have carried out the first study into the Iwasawa-Taniguchi effect for Compton-thick AGN. Our key findings are enumerated below:

(i) We select from the literature a sample of 72 Compton-thick candidate AGN, covering a redshift range of $z \sim 0.0014-3.7$. The candidates were confirmed via an offset between predicted intrinsic and observed X-ray luminosity, given the rest-frame $12 \mu \mathrm{m}$ luminosity interpolated from the Mullaney et al. (2011) AGN infrared spectral template.

(ii) We find an anticorrelation between the rest-frame EW of the narrow core of the neutral $\mathrm{Fe} \mathrm{K} \alpha$ fluorescence emission line and the mid-infrared $12 \mu \mathrm{m}$ continuum luminosity, which we use as a proxy
ID 39: CDFS 384
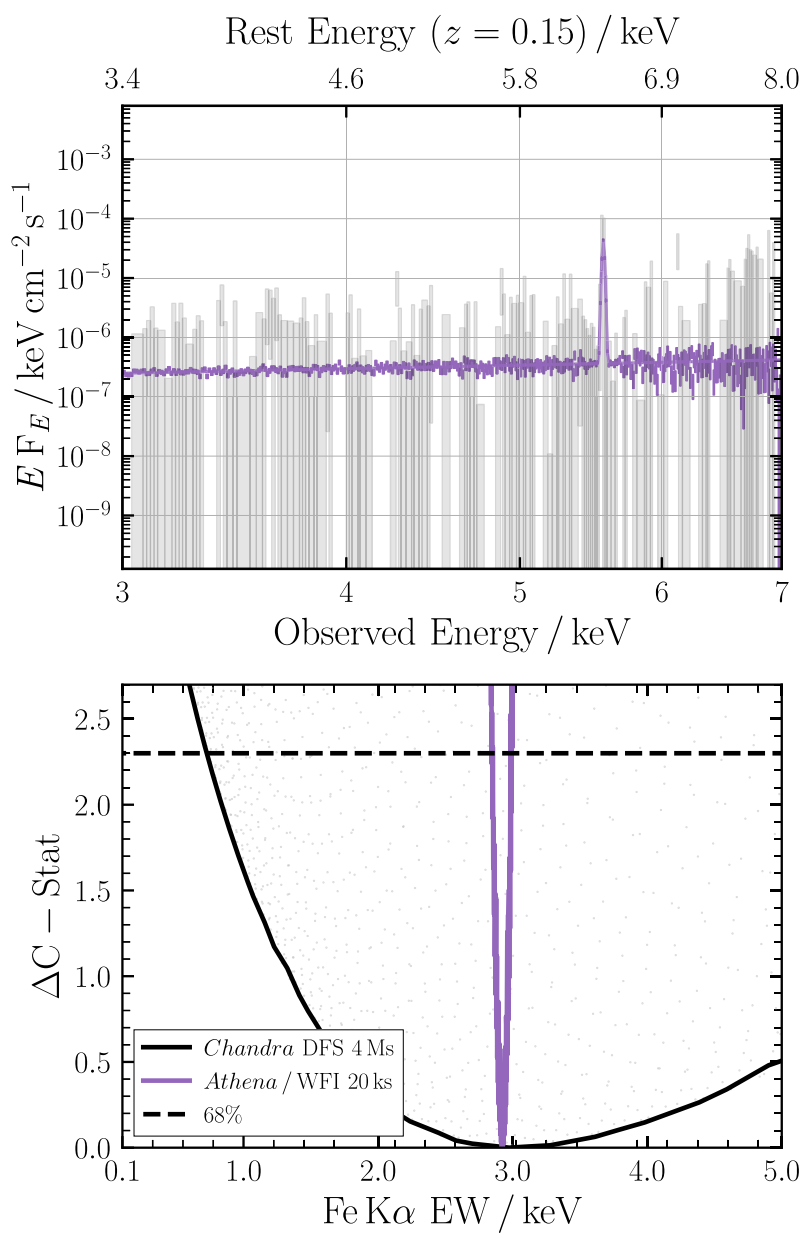

Figure 13. Top panel: Observed CDFS 4 Ms spectrum for CDFS 384 together with simulated Athena/WFI spectrum for a 20 ks observation, shown in black and purple, respectively. Lower panel: Reported EW contour for CDFS 384 used in the paper, together with the improved contour attained with the simulated Athena/WFI spectrum shown above. A dramatic improvement to the $\mathrm{S} / \mathrm{N}$ of the observed spectrum is clearly attainable with Athena, not to mention the improved EW confidence region. Such observations of Compton-thick AGN are to be carried out as part of the Athena mission, with the aim to study obscured accretion and galaxy formation with the WFI instrument.

for the bolometric AGN luminosity. From the Spearman's Rank, we find the anticorrelation to be significant to 98.7 per cent confidence.

(iii) We discuss four possible interpretations of such an anticorrelation:

(a) A luminosity-dependent covering factor (Section 5.2.1).

(b) Luminosity-dependent ionization state of the circumnuclear reprocessing material (Section 5.2.2).

(c) Other possibilities including the contribution from two AGN in a dual system (Section 5.2.3).

(iv) Possible implications of the Compton-thick IwasawaTaniguchi effect include the following:

(a) An increased number density of Compton-thick AGN at higher redshifts due to predicted higher intrinsic luminosities (Section 5.3.1).

(b) Current X-ray reprocessing models do not account for this effect, and as such may incorrectly interpret a weak Fe K $\alpha$ line as 
a signature of Compton-thin reprocessing, leading to an underestimation of the true intrinsic luminosity and hence growth rate of X-ray-obscured AGN.

(c) If a luminosity-dependent covering factor can explain the Iwasawa-Taniguchi effect, it would imply that we are still lacking a population of truly reflection-dominated, luminous Compton-thick AGN, since for $N_{\mathrm{H}} \gtrsim 1.5 \times 10^{24} \mathrm{~cm}^{-2}$, the EW of the Fe K $\alpha$ line are predicted to always be $>1 \mathrm{keV}$, contrary to what we find.

(v) This work further illustrates why the $\mathrm{Fe} \mathrm{K} \alpha$ line alone cannot be directly used to accurately determine the line-of-sight column density to a source. Future dedicated studies of Compton-thick AGN over broad redshift ranges are required to be able to confirm this effect, which could further hold the answers to understanding the physical geometry and evolution of obscuration surrounding AGN.

\section{ACKNOWLEDGEMENTS}

We thank the anonymous referee for invaluable comments on the paper.

P. B. and P. G. (grant reference ST/J003697/2) thank the STFC for support. In addition, the authors thank R. Gilli and C. Circosta for providing the 4 and 7 Msspectra of LESS J0033229.4-275619 used in Fig. 2.

This work was supported in part by the Black Hole Initiative at Harvard University, which is funded by a grant from the John Templeton Foundation.

M. B. acknowledges support from NASA Headquarters under the NASA Earth and Space Science Fellowship Program, grant NNX14AQ07H.

We acknowledge financial support from FONDECYT 1141218 (C. R.), Basal-CATA PFB-06/2007 (C. R.), the China-CONICYT fund (C. R.). This work is partly sponsored by the Chinese Academy of Sciences (CAS), through a grant to the CAS South America Center for Astronomy (CASSACA) in Santiago, Chile.

The scientific results reported in this article are based on observations made by the Chandra X-ray Observatory.

This research has made use of data, software and/or web tools obtained from the High Energy Astrophysics Science Archive Research Center (HEASARC), a service of the Astrophysics Science Division at NASA/GSFC and of the Smithsonian Astrophysical Observatory's High Energy Astrophysics Division.

This research has made use of the NASA/IPAC Extragalactic Database (NED), which is operated by the Jet Propulsion Laboratory, California Institute of Technology, under contract with the National Aeronautics and Space Administration.

This work made use of data from the NUSTAR mission, a project led by the California Institute of Technology, managed by the Jet Propulsion Laboratory, and funded by the National Aeronautics and Space Administration. We thank the NUSTAR Operations, Software and Calibration teams for support with the execution and analysis of these observations. This research has made use of the NuSTAR Data Analysis Software (NUSTARDAS) jointly developed by the ASI Science Data Center (ASDC, Italy) and the California Institute of Technology (USA).

This work is based [in part] on observations made with the Spitzer Space Telescope, which is operated by the Jet Propulsion Laboratory, California Institute of Technology under a contract with NASA.

This publication makes use of data products from the Widefield Infrared Survey Explorer, which is a joint project of the University of California, Los Angeles, and the Jet Propulsion Labo-
ratory/California Institute of Technology, funded by the National Aeronautics and Space Administration. This work made use of the NUMPY (Van Der Walt, Colbert \& Varoquaux 2011), матPLOTLIB (Hunter 2007), SCIPY (Jones et al. 2001), PANDAS (McKinney2010), ASTROPY (Astropy Collaboration 2013) and AdJustTeXT ${ }^{12}$ Python packages.

P. B. would also like to thank S. Hönig, C. Knigge, J. Matthews, M. Middleton, M. Smith, A. Beri, A. Hill, J. Buchner and others for vital scientific discussions into the data analysis and interpretations of the Compton-thick Iwasawa-Taniguchi effect.

\section{REFERENCES}

Annuar A. et al., 2015, ApJ, 815, 36

Annuar A. et al., 2017, ApJ, 836, 165

Antonucci R., 1993, ARA\&A, 31, 473

Arévalo P. et al., 2014, ApJ, 791, 81

Asmus D., Gandhi P., Hönig S. F., Smette A., Duschl W. J., 2015, MNRAS, 454,766

Asmus D., Hönig S. F., Gandhi P., Smette A., Duschl W. J., 2014, MNRAS, 439, 1648

Astropy Collaboration, 2013, A\&A, 558, A33

Baldwin J. A., 1977, ApJ, 214, 679

Ballantyne D. R., 2014, MNRAS, 437, 2845

Baloković M. et al., 2014, ApJ, 794, 111

Baloković M. et al., 2018, ApJ, 854, 42

Bauer F. E. et al., 2015, ApJ, 812, 116

Bianchi S., Guainazzi M., Matt G., Fonseca Bonilla N., 2007, A\&A, 467, L19

Boorman P. G. et al., 2016, ApJ, 833, 245

Brightman M., Nandra K., 2011, MNRAS, 414, 3084

Brightman M., Nandra K., Salvato M., Hsu L.-T., Aird J., Rangel C., 2014, MNRAS, 443, 1999

Brightman M. et al., 2013, MNRAS, 433, 2485

Brightman M. et al., 2016, ApJ, 826, 93

Buchner J. et al., 2015, ApJ, 802, 89

Burlon D., Ajello M., Greiner J., Comastri A., Merloni A., Gehrels N., 2011, ApJ, 728, 58

Cash W., 1979, ApJ, 228, 939

Chen C.-T. J. et al., 2017, ApJ, 837, 145

Corral A. et al., 2016, A\&A, 592, A109

Dewangan G. C., 2002, ApJ, 581, L71

Dey A. et al., 2008, ApJ, 677, 943

Draine B. T., 2003, ApJ, 598, 1026

Farrah D. et al., 2016, ApJ, 831, 76

Feruglio C., Daddi E., Fiore F., Alexander D. M., Piconcelli E., Malacaria C., 2011, ApJ, 729, L4

Gandhi P., Horst H., Smette A., Hönig S., Comastri A., Gilli R., Vignali C., Duschl W., 2009, A\&A, 502, 457

Gandhi P., Hönig S. F., Kishimoto M., 2015b, ApJ, 812, 113

Gandhi P., Yamada S., Ricci C., Asmus D., Mushotzky R. F., Ueda Y., Terashima Y., La Parola V., 2015a, MNRAS, 449, 1845

Gandhi P. et al., 2014, ApJ, 792, 117

Gandhi P. et al., 2017, MNRAS, 467, 4606

García J., Dauser T., Reynolds C. S., Kallman T. R., McClintock J. E., Wilms J., Eikmann W., 2013, ApJ, 768, 146

Georgakakis A. et al., 2017, MNRAS, 469, 3232

Georgantopoulos I. et al., 2013, A\&A, 555, A43

Gilli R. et al., 2011, ApJ, 730, L28

Gilli R. et al., 2014, A\&A, 562, A67

Gohil R., Ballantyne D. R., 2015, MNRAS, 449, 1449

Goulding A. D., Alexander D. M., Bauer F. E., Forman W. R., Hickox R. C., Jones C., Mullaney J. R., Trichas M., 2012, ApJ, 755, 5

${ }^{12}$ https://github.com/Phlya/adjustText 
Haardt F., Maraschi L., 1991, ApJ, 380, L51

Haardt F., Maraschi L., 1993, ApJ, 413, 507

Harrison F. A. et al., 2013, ApJ, 770, 103

Hitomi Collaboration, 2017, preprint (arXiv:1711.06289)

Hlavacek-Larrondo J. et al., 2017, MNRAS, 464, 2223

Hopkins P. F., Hernquist L., Cox T. J., Kereš D., 2008, ApJS, 175, 356

Horst H., Gandhi P., Smette A., Duschl W. J., 2008, A\&A, 479, 389

Hunter J. D., 2007, Comput. Sci. Eng., 9, 90

Ikeda S., Awaki H., Terashima Y., 2009, ApJ, 692, 608

Iwasawa K., Sanders D. B., Evans A. S., Mazzarella J. M., Armus L., Surace J. A., 2009, ApJ, 695, L103

Iwasawa K., Taniguchi Y., 1993, ApJ, 413, L15

Iwasawa K. U V., Mazzarella J. M., Medling A. M., Sanders D. B., Evans A. S., 2018, A\&A, 611, A71

Jiang P., Wang J. X., Wang T. G., 2006, ApJ, 644, 725

Jones E. et al., 2001, SciPy: Open source scientific tools for Python, http: //www.scipy.org/

Kharb P., Lal D. V., Merritt D., 2017, Nat. Astron., 1, 727

Koss M. et al., 2017, ApJ, 850, 74

Koss M. J. et al., 2016, ApJ, 824, L4

Krolik J. H., Madau P., Zycki P. T., 1994, ApJ, 420, L57

Lacy M., Petric A. O., Sajina A., Canalizo G., Storrie-Lombardi L. J., Armus L., Fadda D., Marleau F. R., 2007, AJ, 133, 186

Lanzuisi G., Piconcelli E., Fiore F., Feruglio C., Vignali C., Salvato M., Gruppioni C., 2009, A\&A, 498, 67

Lanzuisi G. et al., 2015, A\&A, 573, A137

Lawrence A., 1991, MNRAS, 252, 586

Lawrence A., Elvis M., 1982, ApJ, 256, 410

Lawrence A., Elvis M., 2010, ApJ, 714, 561

Levenson N. A., Radomski J. T., Packham C., Mason R. E., Schaefer J. J., Telesco C. M., 2009, ApJ, 703, 390

Lightman A. P., White T. R., 1988, ApJ, 335, 57

Lusso E. et al., 2013, ApJ, 777, 86

Lutz D., Maiolino R., Spoon H. W. W., Moorwood A. F. M., 2004, A\&A, 418,465

Lu Y., Yu Q., 1999, ApJ, 526, L5

Malizia A., Molina M., Bassani L., Stephen J. B., Bazzano A., Ubertini P., Bird A. J., 2014, ApJ, 782, L25

Marchesi S., Ajello M., Comastri A., Cusumano G., La Parola V., Segreto A., 2017, ApJ, 836, 116

Masini A, et al., 2017, A\&A, 597, A100

Masini A. et al., 2016, A\&A, 589, A59

Mateos S. et al., 2012, MNRAS, 426, 3271

Mateos S. et al., 2015, MNRAS, 449, 1422

Mateos S. et al., 2017, ApJ, 841, L18

McKinney W., 2010, in van der Walt S., Millman J., eds, Proceedings of the 9th Python in Science Conference. p. 51.

Merloni A. et al., 2014, MNRAS, 437, 3550

Mullaney J. R., Alexander D. M., Goulding A. D., Hickox R. C., 2011, MNRAS, 414, 1082

Murphy K. D., Yaqoob T., 2009, MNRAS, 397, 1549

Nandra K. et al., 2013, preprint (arXiv:1306.2307)
Netzer H., 2015, ARA\&A, 53, 365

Page K. L., O’Brien P. T., Reeves J. N., Turner M. J. L., 2004, MNRAS, 347,316

Planck Collaboration XVI, 2014, A\&A, 571, A16

Polletta M. et al., 2007, ApJ, 663, 81

Ptak A. et al., 2015, ApJ, 800, 104

Puccetti S. et al., 2014, ApJ, 793, 26

Reynolds C. S., 1999, in Poutanen J., Svensson R., eds, ASP Conf. Ser. Vol. 161, High Energy Processes in Accreting Black Holes. Astron. Soc. Pac., San Francisco. p. 178

Ricci C., Paltani S., Awaki H., Petrucci P.-O., Ueda Y., Brightman M., 2013b, A\&A, 553, A29

Ricci C., Paltani S., Ueda Y., Awaki H., 2013a, MNRAS, 435, 1840

Ricci C., Ueda Y., Koss M. J., Trakhtenbrot B., Bauer F. E., Gandhi P., 2015 , ApJ, 815, L13

Ricci C., Ueda Y., Paltani S., Ichikawa K., Gandhi P., Awaki H., 2014, MNRAS, 441, 3622

Ricci C. et al., 2016, ApJ, 820, 5

Ricci C. et al., 2017, ApJ, 835, 105

Risaliti G., Elvis M., Fabbiano G., Baldi A., Zezas A., Salvati M., 2007, ApJ, 659, L111

Risaliti G., Maiolino R., Salvati M., 1999, ApJ, 522, 157

Risaliti G., Young M., Elvis M., 2009, ApJ, 700, L6

Rivers E. et al., 2015, ApJ, 815, 55

Shemmer O., Brandt W. N., Netzer H., Maiolino R., Kaspi S., 2006, ApJ, 646, L29

Shu X. W., Wang J. X., Yaqoob T., Jiang P., Zhou Y. Y., 2012, ApJ, 744, L21

Stern D., 2015, ApJ, 807, 129

Stern D. et al., 2012, ApJ, 753, 30

Teng S. H. et al., 2014, ApJ, 785, 19

Trakhtenbrot B., et al., 2017, MNRAS, 470, 800

Ueda Y. et al., 2011, PASJ, 63, S937

Urry C. M., Padovani P., 1995, PASP, 107, 803

Van Der Walt S., Colbert S. C., Varoquaux G., 2011, CSE, 13, 22

Vito F., Gilli R., Vignali C., Comastri A., Brusa M., Cappelluti N., Iwasawa K., 2014, MNRAS, 445, 3557

Vito F. et al., 2018, MNRAS, 474, 4528

Wright E. L. et al., 2010, AJ, 140, 1868

Wu J. et al., 2012, ApJ, 756, 96

\section{SUPPORTING INFORMATION}

Supplementary data are available at MNRAS online.

Please note: Oxford University Press is not responsible for the content or functionality of any supporting materials supplied by the authors. Any queries (other than missing material) should be directed to the corresponding author for the article.

This paper has been typeset from a $\mathrm{T}_{\mathrm{E}} \mathrm{X} / \mathrm{LT} \mathrm{E} \mathrm{X}$ file prepared by the author. 